\title{
OPTIMAL CHATTERING CONTROLS FOR VISCOUS FLOW
}

\author{
H. O. FATTORINI*and S. S. SRITHARAN ${ }^{\dagger}$ \\ Department of Mathematics, University of California-Los Angeles \\ Los Angeles, CA 90024-1555
}

\begin{abstract}
In this paper we investigate the chattering regimes for a class of optimal control problems in viscous incompressible flow. A typical example involving distributed boundary forcing and nonconvex cost is formulated. Existence of optimal chattering control is established; the control takes values in the space of finitely additive probability measures defined on the control set. Another main result is an approximation of the optimal chattering control by a sequence of suboptimal ordinary controls.
\end{abstract}

\section{Contents}

\section{Introduction}

Optimal control theory of viscous flow has many important applications in engineering science. During the past few years several fundamental advances have been reported for flow control problems with convex cost. Main questions addressed were existence theorem for optimal control [?,?,?,?], necessary conditions for free terminal state problem $[?, ?, ?, ?]$ as well as the full

\footnotetext{
${ }^{*}$ Supported by the NSF under grant DMS-9001793

${ }^{\dagger}$ Mailing address: Code 574, NCCOSC, San Diego, CA 92152-5000; Supported by the ONR Grant No-N00014-91-J-4037
} 
Pontryagin maximum principle for problems with terminal constraint [?] and feedback synthesis using Hamilton-Jacobi -Bellman equation [?,?,?]. Finite element methods for the maximum principle with free end state are analyzed in [?]. See also the forthcoming book [?] for reports of progress by various authors of this field.

In this paper we will study a class of flow control problems where the fluid is controlled by a distributed forcing at a portion of the boundary and the cost functional is nonconvex with respect to the control variable. The fundamental problem of existence of optimal control is resolved by a suitable generalization of the classical concept of Young measures. Young measures were introduced in [?,?] (see also [?]) to deal with nonconvex problems in calculus of variations. During the sixties, several authors applied similar ideas to finite dimensional nonconvex control problems [?,?,?,?,?]. For other applications and theoretical developments see [?,?]. Most of these works are concerned with Young measures defined on finite dimensional sets. However, for the control problems of this paper we need to define probability measures on control sets which are infinite dimensional.

The probability measures used in this paper are of finitely additive type in contrast to the sigma (countably) additive Young measures. When the control set is finite dimensional and bounded, these new measures coincide with the classical Young measures. These measures were first introduced in $[?, ?]$ for semilinear evolution problems with bounded controls.

In $\S 2$ we will formulate a nonconvex control problem in viscous incompressible flow. Here we encounter three features which were not addressed in the previous papers:

(i) Nonconvexity of the control set.

(ii) Nonconvexity of the cost functional with respect to the control variable.

(iii) Nonlinearity of the evolution equation with respect to the control variable.

In $\S 3$ we formulate a general class of infinite dimensional nonlinear control problem based on the properties of various operators that arise in the example formulated in $\S 2$. The solvability theorem proved in this section defines the trajectory for the nonlinear evolution system with respect to ordinary controls. We will show later in $\$ 4$ that the same theorem can be used to deduce the solvability of the relaxed evolution system with chattering (probability measure valued) controls. 
In $\$ 4$ we characterize the space of chattering controls and formulate the relaxed control system. The relaxed control system is obtained by integrating the evolution equation and the cost functional with respect to the "finitely additive Young measures" over the control set. In fact, in this framework, ordinary controls can be viewed as Dirac measures concentrated on individual control curves. In the traditional setting Young measures are chosen with suitable "tightness" to prevent loss of mass under weak limits. In our case loss of mass is prevented by another condition on the control measures. However, tightness is also introduced so that we can integrate unbounded continuous functions with respect to these measures.

In $\$ 5$ we will prove the existence of optimal chattering controls for the relaxed control system. Minimizing sequences of controls will be assumed to satisfy a "de la Vallée Poussin" type [?] criterion. This kind of uniform integrability is also used in the literature on classical Young measures [?,?].

In $\S 6$ we will prove that chattering controls can be approximated by a sequence of ordinary controls in a suitable weak-star topology.

\section{Formulation of nonconvex flow control prob- lems}

Let us consider the task of driving the flow field of a viscous incompressible fluid inside a bounded container as close as possible to a desired velocity field $\boldsymbol{u}_{d}(x, t)$ by suction and blowing at the boundary (for more information on experimental and physical aspects of such control techniques see for example $[?, ?, ?])$. Let $\Omega \subset \boldsymbol{R}^{n}, n=2$ or 3 be a bounded multiply connected open set with $C^{4}$-boundary $\partial \Omega$. We will suppose that $\partial \Omega=\bigcup_{i=1}^{m} \partial \Omega_{i}$ where $\partial \Omega_{i}, i=$ $1, \cdots, m$ are disconnected components of $\partial \Omega$. Let us denote by $(\boldsymbol{u}, p): \Omega \times$ $[0, T] \rightarrow \boldsymbol{R}^{n} \times \boldsymbol{R}$ the velocity and pressure fields. The Navier-Stokes problem is

$$
\begin{array}{lrr}
\boldsymbol{u}_{t}+\boldsymbol{u} \cdot \nabla \boldsymbol{u}=-\nabla p+\nu \Delta \boldsymbol{u} & \text { in } \Omega \times(0, T), \\
\nabla \cdot \boldsymbol{u}=0 & \text { in } \Omega \times(0, T), & \\
\boldsymbol{u}(x, 0)=\boldsymbol{u}_{0}(x), & x \in \Omega,
\end{array}
$$

and 


$$
\boldsymbol{u}(x, t)=\boldsymbol{g}(x, t), \quad(x, t) \in \partial \Omega \times(0, T)
$$

with

$$
\int_{\partial \Omega_{i}} \boldsymbol{g}(x, t) \cdot \boldsymbol{n d} S=0, \quad i=1, \cdots, m \text { and } \quad t \in(0, T) .
$$

Here $\boldsymbol{g}(x, t)$ represents suction and blowing at the boundary, $\boldsymbol{u}_{0}$ is the initial velocity field and $\nu$ the coefficient of kinematic viscosity. We note here that (??) is slightly stronger than the condition $\sum_{i=1}^{m} \int_{\partial \Omega_{i}} \boldsymbol{g}(x, t) \cdot \boldsymbol{n} d S=0$, as dictated by (??).

Let us assume that the desired velocity $\boldsymbol{u}_{d}(x, t)$ be sufficiently smooth. For $\Omega \subset \boldsymbol{R}^{2}$, it is known that [?] if $\boldsymbol{u}_{d}(x, t)$ is a solution orbit on a functional attractor associated with Navier-Stokes system then $\boldsymbol{u}_{d}(x, t)$ would be infinitely smooth in space and time. The optimal control problem is to find the boundary velocity distribution $\boldsymbol{g}(x, t)$ and its time derivative $\boldsymbol{g}_{t}(x, t)$ such that the cost (for example)

$$
2 \nu \int_{0}^{t}\left\|\nabla \boldsymbol{u}_{d}(\cdot, r)-\nabla \boldsymbol{u}(\cdot, r)\right\|_{L^{2}(\Omega)}^{2} d r+\int_{0}^{t} \Upsilon\left(\boldsymbol{g}, \boldsymbol{g}_{r}\right) d r \rightarrow \text { infimum. }
$$

Here the first term contains the energy dissipation of the difference vectorfield and the second term represents the cost of forcing. For more details on the physical aspects of such cost functionals in various fluid flow situations see $[?, ?, ?]$. All the literature quoted earlier dealt with the special case where $\Upsilon=\Upsilon\left(\boldsymbol{g}_{t}\right)$ with $\Upsilon(\cdot)$ convex. Since the function $\Upsilon(\cdot)$ is determined by engineering requirements (and limitations) it is desirable to develop a theory without the convexity assumption. In this paper we will not restrict ourselves to the convex case.

Let us first convert this control problem to an infinite dimensional problem in a suitable Hilbert space. The first step is to construct an extension of the boundary velocity distribution. Our construction method has its roots in the early works of [?], [?] and [?]. We will however use the following sharp result due to [?] (see also [?] for some additional details.). This result provides us an extension operator $\Lambda_{\delta}$ which maps a class of vector fields defined on $\partial \Omega$ into a class of divergence free vector fields defined in $\Omega$. Let us denote by

$$
\dot{H}^{3 / 2}(\partial \Omega):=\left\{\boldsymbol{u} \in H^{3 / 2}(\partial \Omega) ; \quad \int_{\partial \Omega_{i}} \boldsymbol{u} \cdot \boldsymbol{n} d S=0, \quad i=1, \cdots, m\right\}
$$




$$
b(\boldsymbol{u}, \boldsymbol{v}, \boldsymbol{w}):=\sum_{i j=1}^{n} \int_{\Omega} u_{i} \frac{\partial v_{j}}{\partial x^{i}} w_{j} d \boldsymbol{x}
$$

and

$$
\boldsymbol{X}_{1 / 2}:=\left\{\boldsymbol{u} \in H_{0}^{1}(\Omega) ; \quad \nabla \cdot \boldsymbol{u}=0\right\} .
$$

We then have

Proposition $1 \forall \delta>0$, there exists a linear continuous map $\Lambda_{\delta}: \dot{H}^{3 / 2}(\partial \Omega) \rightarrow$ $H^{2}(\Omega)$ such that, $\forall \boldsymbol{\phi} \in \dot{H}^{3 / 2}(\partial \Omega)$,

$$
\begin{array}{lr}
\nabla \cdot \Lambda_{\delta} \boldsymbol{\phi}=0, & \text { in } \Omega, \\
\gamma_{0} \Lambda_{\delta} \boldsymbol{\phi}=\boldsymbol{\phi} & \text { on } \partial \Omega,
\end{array}
$$

where $\gamma_{0}$ is the trace operator. Moreover

$$
\left|b\left(\boldsymbol{v}, \Lambda_{\delta} \boldsymbol{\phi}, \boldsymbol{v}\right)\right| \leq \delta\|\boldsymbol{\phi}\|_{H^{3 / 2}(\partial \Omega)}\|\boldsymbol{v}\|_{H^{1}(\Omega)}^{2}, \quad \forall \boldsymbol{v} \in \boldsymbol{X}_{1 / 2} .
$$

Let us now return to the Navier-Stokes problem (??)-(??) and write the total velocity field as $\boldsymbol{u}(x, t)=\boldsymbol{v}(x, t)+\left[\Lambda_{\delta} \boldsymbol{g}\right](x, t)$. We will then get the following problem for $\boldsymbol{v}$ with homogeneous boundary condition,

$$
\begin{array}{lc}
\boldsymbol{v}_{t}+\boldsymbol{v} \cdot \nabla \boldsymbol{v}+\Lambda_{\delta} \boldsymbol{g} \cdot \nabla \boldsymbol{v}+\boldsymbol{v} \cdot \nabla\left(\Lambda_{\delta} \boldsymbol{g}\right)=-\nabla p+\nu \Delta \boldsymbol{v} \\
+\nu \Delta\left(\Lambda_{\delta} \boldsymbol{g}\right)-\Lambda_{\delta} \boldsymbol{g} \cdot \nabla\left(\Lambda_{\delta} \boldsymbol{g}\right)-\partial_{t}\left(\Lambda_{\delta} \boldsymbol{g}\right) & \text { in } \Omega \times(0, T), \\
\nabla \cdot \boldsymbol{v}=0 & \text { in } \Omega \times(0, T), \\
\boldsymbol{v}(x, t)=0 & \text { on } \partial \Omega \times(0, T)
\end{array}
$$

and

$$
\boldsymbol{v}(x, 0)=\boldsymbol{v}_{0}(x), \quad x \in \Omega .
$$

The cost functional can also be rewritten in terms of the new variables. Let us now apply the standard Hodge projection [?], $P_{H}: L^{2}(\Omega) \rightarrow \boldsymbol{X}$ where

$$
\boldsymbol{X}:=\left\{\boldsymbol{u} \in L^{2}(\Omega) ; \nabla \cdot \boldsymbol{u}=0,\left.\boldsymbol{u} \cdot \boldsymbol{n}\right|_{\partial \Omega}=0\right\}
$$

to the above system (??)-(??) to get, 


$$
\begin{aligned}
& \boldsymbol{v}_{t}+\mathcal{A} \boldsymbol{v}+\mathcal{B}(\boldsymbol{v}, \boldsymbol{v})+\mathcal{B}\left(\Lambda_{\delta} \boldsymbol{g}, \boldsymbol{v}\right)+\mathcal{B}\left(\boldsymbol{v}, \Lambda_{\delta} \boldsymbol{g}\right) \\
& +\mathcal{B}\left(\Lambda_{\delta} \boldsymbol{g}, \Lambda_{\delta} \boldsymbol{g}\right)+\mathcal{L} \Lambda_{\delta} \boldsymbol{g}+P_{H} \partial_{t}\left(\Lambda_{\delta} \boldsymbol{g}\right)=0
\end{aligned}
$$

Here we denote

$$
\mathcal{A} \boldsymbol{v}:=-\nu P_{H} \Delta \boldsymbol{v}, \quad \forall \boldsymbol{v} \in H^{2}(\Omega) \cap \boldsymbol{X}_{1 / 2}
$$

and

$$
\mathcal{L} \boldsymbol{w}:=-\nu P_{H} \Delta \boldsymbol{w}, \quad \forall \boldsymbol{w} \in H^{2}(\Omega) .
$$

$\mathcal{A}$ is the well known Stokes operator and $\mathcal{B}(\cdot, \cdot)$ is the standard bilinear operator which is associated with the inertia term in Navier-Stokes theory.

Let us now take the forcing on the boundary with the following properties,

$$
\boldsymbol{g}(x, t)=\boldsymbol{g}_{i}(x, t), \quad(x, t) \in \partial \Omega_{i} \times(0, T), i=1, \cdots, m
$$

with supports,

$$
\text { Supp } \boldsymbol{g}_{i} \subset \partial \Omega_{i}, \quad i=1, \cdots, m .
$$

Equation (??) implies

$$
\int_{\partial \Omega_{i}} \boldsymbol{g}_{i}(x, t) \cdot \boldsymbol{n} d S=0, \quad i=1, \cdots, m .
$$

If $\delta$ is chosen small enough, we can ensure that

$$
\operatorname{Supp} \Lambda_{\delta} \boldsymbol{g}_{i} \cap \operatorname{Supp} \Lambda_{\delta} \boldsymbol{g}_{j}=\emptyset, \quad \text { for } i \neq j \text {. }
$$

We can thus write $\Lambda_{\delta} \boldsymbol{g}(x, t)=\sum_{i=1}^{m} \Lambda_{\delta} \boldsymbol{g}_{i}(x, t)$. In our earlier papers we analyzed a special form of the above forcing where $\boldsymbol{g}_{i}(x, t)=\boldsymbol{g}_{i}(x) l_{i}(t)$ with $\boldsymbol{g}_{i}(x)$ a prescribed function. In this case the control set would be finite dimensional. In this paper, however, we will not consider such restrictions.

Let us take the control as

$$
\begin{aligned}
& U(t)=\left(U_{11}(t), \cdots, U_{1 m}(t), U_{21}(t), \cdots, U_{2 m}(t)\right) \\
& :=\left(\Lambda_{\delta} \boldsymbol{g}_{1}(\cdot, t), \cdots, \Lambda_{\delta} \boldsymbol{g}_{m}(\cdot, t), \partial_{t} \Lambda_{\delta} \boldsymbol{g}_{1}(\cdot, t), \cdots, \partial_{t} \Lambda_{\delta} \boldsymbol{g}_{m}(\cdot, t)\right) .
\end{aligned}
$$

Note that with this definition, for a given $t$, each $U_{i j}(t)$ takes its value in an infinite dimensional space. We now rewrite (??) as,

$$
\boldsymbol{v}_{t}+\mathcal{A} \boldsymbol{v}+\mathcal{B}(\boldsymbol{v})=\mathcal{N}(\boldsymbol{v}, U),
$$

where $\mathcal{B}(\boldsymbol{v}):=\mathcal{B}(\boldsymbol{v}, \boldsymbol{v})$ and

$$
\mathcal{N}(\boldsymbol{v}, U):=-\sum_{i=1}^{m}\left\{\mathcal{B}\left(U_{1 i}, \boldsymbol{v}\right)+\mathcal{B}\left(\boldsymbol{v}, U_{1 i}\right)+\mathcal{B}\left(U_{1 i}, U_{1 i}\right)+\mathcal{L} U_{1 i}+P_{H} U_{2 i}\right\}
$$




\section{Mathematical formulation and definition of trajectories}

We consider the control system

$$
\begin{aligned}
& \boldsymbol{v}_{t}+\mathcal{A} \boldsymbol{v}+\mathcal{B}(\boldsymbol{v})=\mathcal{N}(\boldsymbol{v}, U), \quad t \in(0, T), \\
& \boldsymbol{v}_{0}=\boldsymbol{\zeta} \in \boldsymbol{X}
\end{aligned}
$$

in the Hilbert space $\boldsymbol{X}$ with scalar product $(\cdot, \cdot)$ and norm $\|\cdot\|$. The Stokes operator $\mathcal{A}$ satisfies the following well known properties [?].

Proposition $2 \mathcal{A}$ is self-adjoint, positive definite and $D(\mathcal{A})=H^{2}(\Omega) \cap$ $\boldsymbol{X}_{1 / 2}$.

These results have the following consequences. $-\mathcal{A}$ generates a holomorphic semigroup $S(t)=\exp (-t \mathcal{A})$. The fractional powers $\mathcal{A}^{\alpha}, \alpha \in \boldsymbol{R}$ are well defined and $\mathcal{A}^{\alpha}$ for $\alpha \leq 0$ are bounded. For $\alpha \geq 0$ we write $\boldsymbol{X}_{\alpha}=D\left(\mathcal{A}^{\alpha}\right)$ and equip this space with the natural inner product $(\boldsymbol{v}, \boldsymbol{u})_{\alpha}=\left(\mathcal{A}^{\alpha} \boldsymbol{v}, \mathcal{A}^{\alpha} \boldsymbol{u}\right)$, corresponding to the norm $\|\boldsymbol{v}\|_{\alpha}=\left\|\mathcal{A}^{\alpha} \boldsymbol{v}\right\|$. The particular cases $\alpha=0$ and $1 / 2$ are also given by (??) and (??) respectively. For $\alpha<0, \boldsymbol{X}_{\alpha}$ is the completion of $\boldsymbol{X}$ under $\|\cdot\|_{\alpha}$.

The inertia term $\mathcal{B}(\cdot)$ satisfies the following

Theorem 1 There exists $\beta, 0 \leq \beta<1 / 2$ such that $\mathcal{B}(\cdot)$ maps $\boldsymbol{X}_{1 / 2}$ into $\boldsymbol{X}_{-\beta}$. Moreover, $\mathcal{B}(\cdot): \boldsymbol{X}_{1 / 2} \rightarrow \boldsymbol{X}_{-\beta}$ is continuous, locally bounded, and locally Lipschitz continuous, i.e. for every $C>0$ there exist constants $K_{B}=$ $K_{B}(C), L_{B}=L_{B}(C)$ such that

$$
\begin{aligned}
& \|\mathcal{B}(\boldsymbol{v})\|_{-\beta} \leq K_{B}, \text { for } \boldsymbol{v} \in \boldsymbol{X}_{1 / 2} \text { and }\|\boldsymbol{v}\|_{1 / 2} \leq C, \\
& \|\mathcal{B}(\boldsymbol{v})-\mathcal{B}(\boldsymbol{u})\|_{-\beta} \leq L_{B}\|\boldsymbol{v}-\boldsymbol{u}\|_{1 / 2}, \\
& \text { for } \boldsymbol{v}, \boldsymbol{u} \in \boldsymbol{X}_{1 / 2} \text { and }\|\boldsymbol{v}\|_{1 / 2},\|\boldsymbol{u}\|_{1 / 2} \leq C
\end{aligned}
$$

This theorem implies that $\mathcal{A}^{-\beta} \mathcal{B}\left(\mathcal{A}^{-1 / 2} \cdot\right)$, as a map from $\boldsymbol{X}$ into $\boldsymbol{X}$, is continuous, locally bounded and locally Lipschitz continuous: for each $C>0$,

$$
\left\|\mathcal{A}^{-\beta} \mathcal{B}\left(\mathcal{A}^{-1 / 2} \boldsymbol{z}\right)\right\| \leq K_{B}, \text { for } \boldsymbol{z} \in \boldsymbol{X} \text { and }\|\boldsymbol{z}\| \leq C
$$




$$
\begin{aligned}
& \left\|\mathcal{A}^{-\beta} \mathcal{B}\left(\mathcal{A}^{-1 / 2} \boldsymbol{z}\right)-\mathcal{A}^{-\beta} \mathcal{B}\left(\mathcal{A}^{-1 / 2} \boldsymbol{w}\right)\right\| \leq L_{B}\|\boldsymbol{z}-\boldsymbol{w}\|, \\
& \text { for } \boldsymbol{z}, \boldsymbol{w} \in \boldsymbol{X} \text { and }\|\boldsymbol{z}\|,\|\boldsymbol{w}\| \leq C
\end{aligned}
$$

Proof: The inertia term $\mathcal{B}(\boldsymbol{v})=\mathcal{B}(\boldsymbol{v}, \boldsymbol{v})$ is defined using the trilinear form (??) as,

$$
b(\boldsymbol{v}, \boldsymbol{u}, \boldsymbol{w})=<\mathcal{B}(\boldsymbol{v}, \boldsymbol{u}), \boldsymbol{w}>.
$$

The following estimate [?] for $b(\cdot, \cdot, \cdot)$ characterizes the properties of $\mathcal{B}(\cdot)$ : if $\Omega \subset \boldsymbol{R}^{n}$, then

$$
\begin{aligned}
& |b(\boldsymbol{u}, \boldsymbol{v}, \boldsymbol{w})| \leq C_{0}\|\boldsymbol{u}\|_{H^{2 \alpha}(\Omega)}\|\boldsymbol{v}\|_{H^{2 \gamma+1}(\Omega)}\|\boldsymbol{w}\|_{H^{2 \beta}(\Omega)}, \\
& \forall \boldsymbol{u} \in H^{2 \alpha}(\Omega), \forall \boldsymbol{v} \in H^{2 \gamma+1}(\Omega), \forall \boldsymbol{u} \in H^{2 \beta}(\Omega)
\end{aligned}
$$

with $\alpha+\beta+\gamma \geq n / 4$ if $\alpha, \beta, \gamma \neq n / 4$ and $\alpha+\beta+\gamma>n / 4$ if one of $\alpha, \beta$ or $\gamma$ coincides with $n / 4$. From this we can also deduce

$$
\begin{aligned}
& |b(\boldsymbol{u}, \boldsymbol{v}, \boldsymbol{w})| \leq C_{0}\|\boldsymbol{u}\|_{\alpha}\|\boldsymbol{v}\|_{\gamma+1 / 2}\|\boldsymbol{w}\|_{\beta}, \\
& \forall \boldsymbol{u} \in \boldsymbol{X}_{\alpha}, \forall \boldsymbol{v} \in \boldsymbol{X}_{\gamma+1 / 2}, \forall \boldsymbol{u} \in \boldsymbol{X}_{\beta} .
\end{aligned}
$$

From (??), taking $\alpha=1 / 2, \gamma=0, \beta>0$ for $n=2$ and $\beta=1 / 4$ for $n=3$, we get,

$$
|b(\boldsymbol{v}, \boldsymbol{v}, \boldsymbol{w})|=|<\mathcal{B}(\boldsymbol{v}), \boldsymbol{w}>| \leq C_{0}\|\boldsymbol{v}\|_{1 / 2}^{2}\|\boldsymbol{w}\|_{\beta}, \forall \boldsymbol{v} \in \boldsymbol{X}_{1 / 2}, \forall \boldsymbol{w} \in \boldsymbol{X}_{\beta} .(36)
$$

This gives (??). Similarly, using the trilinearity of $b(\cdot, \cdot, \cdot)$ we get,

$$
\begin{aligned}
& <\mathcal{B}(\boldsymbol{u})-\mathcal{B}(\boldsymbol{v}), \boldsymbol{w}>=b(\boldsymbol{u}, \boldsymbol{u}, \boldsymbol{w})-b(\boldsymbol{v}, \boldsymbol{v}, \boldsymbol{w}) \\
& =b(\boldsymbol{u}-\boldsymbol{v}, \boldsymbol{u}, \boldsymbol{w})+b(\boldsymbol{v}, \boldsymbol{u}-\boldsymbol{v}, \boldsymbol{w})
\end{aligned}
$$

Thus, again using the same values of $\alpha, \beta, \gamma$ we get

$$
\begin{aligned}
& |<\mathcal{B}(\boldsymbol{u})-\mathcal{B}(\boldsymbol{v}), \boldsymbol{w}>| \leq C_{0}\left\{\|\boldsymbol{u}\|_{1 / 2}+\|\boldsymbol{v}\|_{1 / 2}\right\}\|\boldsymbol{u}-\boldsymbol{v}\|_{1 / 2}\|\boldsymbol{w}\|_{\beta}, \\
& \forall \boldsymbol{u} \in \boldsymbol{X}_{1 / 2}, \forall \boldsymbol{v} \in \boldsymbol{X}_{1 / 2}, \forall \boldsymbol{w} \in \boldsymbol{X}_{\beta} .
\end{aligned}
$$

From this we obtain (??). 
The control $U(\cdot)$ defined in (??), takes its values in the control set $\boldsymbol{U}$ which is defined as an arbitrary subset $\boldsymbol{U} \subset\left(H^{2}(\Omega)\right)^{\otimes m} \times\left(L^{2}(\Omega)\right)^{\otimes m}$. The control operator $\mathcal{N}(\boldsymbol{v}, U)$ is defined in $\boldsymbol{X}_{1 / 2} \times \boldsymbol{U}$.

Theorem $2 \mathcal{N}(\cdot, \cdot)$ continuously maps $\boldsymbol{X}_{1 / 2} \times \boldsymbol{U}$ into $\boldsymbol{X}$. There exists a continuous function $\kappa(\cdot): \boldsymbol{U} \rightarrow \boldsymbol{R}, \kappa(U) \geq 1$ such that, for every $C>0$ there exist $K_{N}=K_{N}(C)$ and $L_{N}=L_{N}(C)$ such that

$$
\begin{aligned}
& \|\mathcal{N}(\boldsymbol{v}, U)\| \leq K_{N} \kappa(U), \text { for } \boldsymbol{v} \in \boldsymbol{X}_{1 / 2},\|\boldsymbol{v}\|_{1 / 2} \leq C, U \in \boldsymbol{U}, \\
& \|\mathcal{N}(\boldsymbol{v}, U)-\mathcal{N}(\boldsymbol{u}, U)\| \leq L_{N}\|\boldsymbol{v}-\boldsymbol{u}\|_{1 / 2} \kappa(U), \\
& \text { for } \boldsymbol{v} \in \boldsymbol{X}_{1 / 2}, \boldsymbol{u} \in \boldsymbol{X}_{1 / 2}, U \in \boldsymbol{U} \text { and }\|\boldsymbol{v}\|_{1 / 2},\|\boldsymbol{u}\|_{1 / 2} \leq C .
\end{aligned}
$$

From the form of the operator $\mathcal{N}$ in (??), the definition (??) of the operator $\mathcal{L}$ and the estimate (??) of the trilinear form, Theorem ?? can be easily proved using steps similar to the proof of Theorem ??. A suitable form of $\kappa(\cdot)$ is

$$
\kappa(U)=\sum_{i=1}^{m}\left\{\left\|U_{1 i}\right\|_{H^{2}(\Omega)}^{2}+\left\|U_{1 i}\right\|_{H^{2}(\Omega)}+\left\|U_{2 i}\right\|_{L^{2}(\Omega)}\right\}+1 .
$$

Inequalities (??)-(??) imply corresponding inequalities (in the norm of $\boldsymbol{X}$ ) for the operator $\mathcal{N}\left(\mathcal{A}^{-1 / 2}, \cdot \cdot\right)$, mapping $\boldsymbol{X} \times \boldsymbol{U}$ into $\boldsymbol{X}$ :

$$
\begin{aligned}
& \left\|\mathcal{N}\left(\mathcal{A}^{-1 / 2} \boldsymbol{\psi}, U\right)\right\| \leq K_{N} \kappa(U), \text { for } \boldsymbol{\psi} \in \boldsymbol{X},\|\boldsymbol{\psi}\| \leq C, U \in \boldsymbol{U} . \\
& \left\|\mathcal{N}\left(\mathcal{A}^{-1 / 2} \boldsymbol{\psi}, U\right)-\mathcal{N}\left(\mathcal{A}^{-1 / 2} \boldsymbol{\zeta}, U\right)\right\| \leq L_{N}\|\boldsymbol{\psi}-\boldsymbol{\zeta}\| \kappa(U), \\
& \text { for } \boldsymbol{\psi} \in \boldsymbol{X}, \boldsymbol{\zeta} \in \boldsymbol{X}, U \in \boldsymbol{U} \text { and }\|\boldsymbol{\psi}\|,\|\boldsymbol{\xi}\| \leq C .
\end{aligned}
$$

The space $\mathcal{U}_{\text {ad }}(0, T ; \boldsymbol{U}, \kappa)$ of admissible controls consists of all $\boldsymbol{U}$-valued functions defined almost everywhere and satisfying,

Control Space Hypothesis $1 \kappa(U(\cdot)) \in L^{2}(0, T)$. 
We will see in $\$ 4$ that this condition on the control is in fact the "tightness condition" imposed on the corresponding chattering control (Dirac measure concentrated on this control). Note also that this hypothesis and definitions (??), (??) imply that, $\forall \boldsymbol{v}(\cdot) \in C\left([0, T] ; \boldsymbol{X}_{1 / 2}\right)$, the control operator $\mathcal{N}(\boldsymbol{v}(\cdot), U(\cdot))$ is strongly measurable. In fact, we have $\mathcal{N}(\boldsymbol{v}(\cdot), U(\cdot)) \in$ $L^{2}(0, T ; \boldsymbol{X})$.

By definition, solutions or trajectories of the initial value problem (??)(??) in an interval $0 \leq t \leq T^{\prime}$ are $\boldsymbol{X}_{1 / 2}$-valued functions $\boldsymbol{v}(\cdot)$ continuous in the norm of $\boldsymbol{X}_{1 / 2}$ and satisfying

$$
\begin{aligned}
& \boldsymbol{v}(t)=S(t) \boldsymbol{\zeta}-\int_{0}^{t} \mathcal{A}^{\beta} S(t-r) \mathcal{A}^{-\beta} \mathcal{B}(\boldsymbol{v}(r)) d r \\
& +\int_{0}^{t} S(t-r) \mathcal{N}(\boldsymbol{v}(r), U(r)) d r, \quad 0 \leq t \leq T^{\prime} .
\end{aligned}
$$

We note that, since controls are not smooth, solutions of (??) may fail to be differentiable or to belong to $D(\mathcal{A})$, that is, may not be strong solutions.

The initial value problem with $\mathcal{N}(\boldsymbol{v}, U)=\mathcal{L}_{B} U$ where $\mathcal{L}_{B}$ is a linear bounded operator was studied in [?] and the results there can be extended to the present situation. We transform the integral equation (??) into an integral equation for $\boldsymbol{z}(t)=\mathcal{A}^{1 / 2} \boldsymbol{v}(t)$ :

$$
\begin{aligned}
& \boldsymbol{z}(t)=[\boldsymbol{M} \boldsymbol{z}](t)=\mathcal{A}^{1 / 2} S(t) \boldsymbol{\zeta}-\int_{0}^{t} \mathcal{A}^{1 / 2+\beta} S(t-r) \mathcal{A}^{-\beta} \mathcal{B}\left(\mathcal{A}^{-1 / 2} \boldsymbol{z}(r)\right) d r \\
& +\int_{0}^{t} \mathcal{A}^{1 / 2} S(t-r) \mathcal{N}\left(\mathcal{A}^{-1 / 2} \boldsymbol{z}(r), U(r)\right) d r, \quad 0 \leq t \leq T^{\prime}
\end{aligned}
$$

where the interval $\left[0, T^{\prime}\right]$ is to be determined below. If $\boldsymbol{z}(\cdot)$ is a continuous $\boldsymbol{X}$-valued solution of (??) in $0 \leq t \leq T^{\prime}$, then $\boldsymbol{v}(t)=\mathcal{A}^{-1 / 2} \boldsymbol{z}(t)$ is the required solution of (??). We recall [?] that, for any $\alpha>0$ there exists a constant $C_{\alpha}$ such that

$$
\left\|\mathcal{A}^{\alpha} S(t)\right\| \leq C_{\alpha} t^{-\alpha}, \quad t>0
$$

so that (in the light of (??) the first integral on the right side of (??) makes sense. For the second integral, we use the estimate (??) and the fact [?] that if $\boldsymbol{g}(\cdot) \in L^{2}(a, b ; \boldsymbol{X})$ then the function 


$$
\boldsymbol{y}(t)=\int_{a}^{t} \mathcal{A}^{1 / 2} S(t-r) \boldsymbol{g}(r) d r, \quad a \leq t \leq b
$$

belongs to $C([a, b] ; \boldsymbol{X})$ with

$$
\|\boldsymbol{y}(\cdot)\|_{C([a, b] ; X)} \leq \frac{1}{\sqrt{2}}\|\boldsymbol{g}(\cdot)\|_{L^{2}(a, b ; X)} .
$$

The following results defines the trajectory for our control system.

Lemma 1 Let $\boldsymbol{\zeta} \in \boldsymbol{X}_{1 / 2}$ and $U(\cdot) \in \mathcal{U}_{a d}(0, T ; \boldsymbol{U}, \kappa)$. Then (??) possesses a unique solution $\boldsymbol{v}(\cdot) \in C\left(\left[0, T^{\prime}\right] ; \boldsymbol{X}_{1 / 2}\right)$ for some $T^{\prime} \leq T$ (equivalently, (??) possesses a unique solution $\left.\boldsymbol{z}(\cdot) \in C\left(\left[0, T^{\prime}\right] ; \boldsymbol{X}\right)\right)$.

Proof: We consider (??) in $C\left(\left[0, T^{\prime}\right] ; \boldsymbol{X}\right)$. Let $\boldsymbol{B}\left(S(\cdot) \mathcal{A}^{1 / 2} \boldsymbol{\zeta}, 1\right)$ be the ball of center $S(\cdot) \mathcal{A}^{1 / 2} \boldsymbol{\zeta} \in C\left(\left[0, T^{\prime}\right] ; \boldsymbol{X}\right)$ and radius 1 . If $\boldsymbol{z}(\cdot) \in \boldsymbol{B}\left(S(\cdot) \mathcal{A}^{1 / 2} \boldsymbol{\zeta}, 1\right)$ then using (??), (??) and (??), we have

$$
\begin{aligned}
& \left\|[\boldsymbol{M} \boldsymbol{z}](t)-S(t) \mathcal{A}^{1 / 2} \boldsymbol{\zeta}\right\| \leq C_{1 / 2+\beta} K_{B} \int_{0}^{t}(t-r)^{-1 / 2-\beta} d r \\
& +\frac{K_{N}}{\sqrt{2}}\|\kappa(U(\cdot))\|_{L^{2}\left(0, T^{\prime}\right)} \cdot
\end{aligned}
$$

where $C_{1 / 2+\beta}$ is the constant in (??), $K_{B}$ is a bound for

$$
\left\|\mathcal{A}^{-\beta} \mathcal{B}\left(\mathcal{A}^{-1 / 2} \boldsymbol{z}(t)\right)\right\|, \text { when }\left\|\boldsymbol{z}(t)-S(t) \mathcal{A}^{1 / 2} \boldsymbol{\zeta}\right\| \leq 1, \quad 0 \leq t \leq T
$$

and $K_{N} \kappa(U(t))$ is the corresponding bound for $\left\|\mathcal{N}\left(\mathcal{A}^{-1 / 2} \boldsymbol{z}(t), U(t)\right)\right\|$. Accordingly, choosing $T^{\prime}$ sufficiently small we can insure that $\boldsymbol{M}$ maps $\boldsymbol{B}\left(S(\cdot) \mathcal{A}^{1 / 2} \boldsymbol{\zeta}, 1\right)$ into itself. Using now the local Lipschitz conditions (??) and (??) for the ball $\boldsymbol{B}\left(S(\cdot) \mathcal{A}^{1 / 2} \boldsymbol{\zeta}, 1\right)$ and the estimate (??) we deduce that

$$
\begin{aligned}
& \left\|\left[\boldsymbol{M} \boldsymbol{z}_{1}\right](t)-\left[\boldsymbol{M} \boldsymbol{z}_{2}\right](t)\right\| \\
& \leq C_{1 / 2+\beta} \int_{0}^{t}(t-r)^{-1 / 2-\beta}\left\|\mathcal{A}^{-\beta} \mathcal{B}\left(\mathcal{A}^{-1 / 2} \boldsymbol{z}_{1}(r)\right)-\mathcal{A}^{-\beta} \mathcal{B}\left(\mathcal{A}^{-1 / 2} \boldsymbol{z}_{2}(r)\right)\right\| d r \\
& +\frac{1}{\sqrt{2}}\left\{\int_{0}^{T^{\prime}}\left\|\mathcal{N}\left(\mathcal{A}^{-1 / 2} \boldsymbol{z}_{1}(r), U(r)\right)-\mathcal{N}\left(\mathcal{A}^{-1 / 2} \boldsymbol{z}_{2}(r), U(r)\right)\right\|^{2} d r\right\}^{1 / 2}
\end{aligned}
$$




$$
\begin{aligned}
& \leq C_{1 / 2+\beta} L_{B} \int_{0}^{t}(t-r)^{-1 / 2-\beta}\left\|\boldsymbol{z}_{1}(r)-\boldsymbol{z}_{2}(r)\right\| d r \\
& \left.\left.+\frac{L_{N}}{\sqrt{2}}\left\{\int_{0}^{T^{\prime}} \| \boldsymbol{z}_{1}(r)\right)-\boldsymbol{z}_{2}(r)\right) \|^{2} \kappa(U(r))^{2} d r\right\}^{1 / 2}
\end{aligned}
$$

where $L_{B}, L_{N}$ are the Lipschitz constants corresponding to the ball $\boldsymbol{B}\left(S(\cdot) \mathcal{A}^{1 / 2} \boldsymbol{\zeta}, 1\right)$. It follows easily from (??) that $\boldsymbol{M}$ will be a contraction map in

$$
\boldsymbol{B}\left(S(\cdot) \mathcal{A}^{1 / 2} \boldsymbol{\zeta}, 1\right) \subset C\left(\left[0, T^{\prime}\right] \boldsymbol{X}\right) \text { for } T^{\prime} \text { small enough. }
$$

Applying the contraction mapping principle, we obtain a unique solution $\boldsymbol{z}(t)$ of (??) in $0 \leq t \leq T^{\prime}$ and, setting $\boldsymbol{v}(t)=\mathcal{A}^{-1 / 2} \boldsymbol{z}(t)$, a solution of (??) in the same interval. Let now $\boldsymbol{v}(t)$ be an arbitrary solution of (??) with $\boldsymbol{\zeta} \in D\left(\mathcal{A}^{1 / 2}\right)$. Then $\boldsymbol{z}(t)=\mathcal{A}^{1 / 2} \boldsymbol{v}(t)$ is a solution of the integral equation (??), thus uniqueness of $\boldsymbol{z}(t)$ implies uniqueness of $\boldsymbol{v}(t)$.

The arguments used in the proof of Lemma?? imply that, if $\boldsymbol{v}(t)$ is a solution of (??) in a closed interval $0 \leq t \leq T^{\prime}$, then $\boldsymbol{v}(t)$ can always be extended to a larger interval $0 \leq t \leq T^{\prime \prime}, T^{\prime \prime}>T^{\prime}$ solving the equation in $t \geq T^{\prime}$ with $\boldsymbol{v}\left(T^{\prime}\right)$ as initial condition. This implies that each solution $\boldsymbol{v}(\cdot)$ of $(? ?)$ either exists in $0 \leq t \leq T$ or possesses a maximal interval of existence $\left[0, T_{m}\right), T_{m}<T$ with $\lim \sup _{t \rightarrow T_{m}}\|\boldsymbol{v}(t)\|_{1 / 2}=+\infty$. Hence we have

Lemma 2 Let $\boldsymbol{v}(\cdot)$ be a solution of (??) in the interval $0 \leq t<T^{\prime}$ with $\zeta \in D\left(\mathcal{A}^{1 / 2}\right)$. Suppose that

$$
\|\boldsymbol{v}(t)\|_{1 / 2} \leq C, \quad 0 \leq t<T^{\prime}
$$

Then, if $\left[0, T_{m}\right)$ is the maximal interval of existence of $\boldsymbol{v}(\cdot)$, we have $T_{m}>T^{\prime}$.

We denote by $\boldsymbol{v}(t, U)$ the solution (or trajectory) of (??)-(??) (or, rather, of the integral equation (??)).

The cost functional of the problem is

$$
\mathcal{C}(t, U)=\int_{0}^{t} \Theta(\boldsymbol{v}(r, U), U(r)) d r .
$$


Hypothesis $\mathbf{1} \Theta(\cdot, \cdot)$ maps $\boldsymbol{X}_{1 / 2} \times \boldsymbol{U}$ into $\boldsymbol{R}$ and for every $C>0$ there exists $K_{\Theta}=K_{\Theta}(C)$ such that

$$
|\Theta(\boldsymbol{v}, U)| \leq K_{\Theta} \kappa(U)^{2}, \quad \boldsymbol{v} \in \boldsymbol{X}_{1 / 2}, U \in \boldsymbol{U} \text { and }\|\boldsymbol{v}\|_{1 / 2} \leq C .
$$

When a cost functional is at play, the space $\mathcal{U}_{\mathrm{ad}}(0, T ; \boldsymbol{U}, \kappa)$ is also required to satisfy

Control Space Hypothesis 2 For every $\boldsymbol{v}(\cdot) \in C\left([0, T] ; \boldsymbol{X}_{1 / 2}\right)$ and every $U(\cdot) \in \mathcal{U}_{a d}(0, T ; \boldsymbol{U}, \kappa), \Theta(\boldsymbol{v}(\cdot), U(\cdot))$ is measurable. Moreover, $\kappa(U)^{-2} \Theta(\boldsymbol{v}, U)$ is continuous in $\boldsymbol{X}_{1 / 2} \times \boldsymbol{U}$, uniformly with respect to $\boldsymbol{v}$ for bounded $\boldsymbol{v}$ and $U \in \boldsymbol{U}$.

This implies that $(\boldsymbol{v}, U) \rightarrow \kappa(U)^{-2} \Theta(\boldsymbol{v}, U)$ is jointly continuous and

$$
\sup \left\{\left|\kappa(U)^{-2} \Theta(\boldsymbol{w}, U)-\kappa(U)^{-2} \Theta(\boldsymbol{v}, U)\right| ; U \in \boldsymbol{U}\right\} \rightarrow 0
$$

as $\|\boldsymbol{w}-\boldsymbol{v}\|_{1 / 2} \rightarrow 0$ uniformly for $\boldsymbol{w}, \boldsymbol{v}$ in a bounded set in $\boldsymbol{X}_{1 / 2}$.

This hypothesis insures that $\mathcal{C}(t, U)$ is well defined; in fact, by (??) $\Theta(\boldsymbol{v}(\cdot), U(\cdot)) \in L^{1}(0, T)$.

The optimal control problem for (??)-(??) and (??) consists of characterizing the control $\hat{U}(\cdot) \in \mathcal{U}_{\mathrm{ad}}(0, T ; \boldsymbol{U}, \kappa)$ that minimizes the cost functional $\mathcal{C}(t, U)$ subject to target condition

$$
\boldsymbol{v}(t, U) \in \boldsymbol{Y} \subseteq \boldsymbol{X}
$$

where the closed set $\boldsymbol{Y}$ is called the target set. In the next section we consider a relaxed version of (??),(??),(??) and (??) where the class of admissible controls is considerably enlarged and provides automatic existence theorems for optimal control problems.

\section{Spaces of chattering controls and the re- laxed control system}

We introduce two classes of chattering controls, called

$$
\mathcal{V}_{\mathrm{bc}}(0, T ; \boldsymbol{U}, \kappa) \text { and } \mathcal{V}_{\mathrm{b}}(0, T ; \boldsymbol{U}, \kappa) \text { respectively. }
$$


In the first, the control set $\boldsymbol{U}$ is required to be a normal topological space [?] and the instantaneous values of the chattering controls are regular finitely additive probability measures on $\boldsymbol{U}$. In the second, $\boldsymbol{U}$ is an arbitrary set and the measures are finitely additive probability measures. We shall give full details for $\mathcal{V}_{\mathrm{bc}}(0, T ; \boldsymbol{U}, \kappa)$ and indicate later the modifications that apply to the other class.

Construction of the class $\mathcal{V}_{\mathrm{bc}}(0, T ; \boldsymbol{U}, \kappa)$ is based on the dual of the space $L^{1}(0, T ; \boldsymbol{E})$ of all (equivalence classes of) strongly measurable $\boldsymbol{E}$-valued functions $\boldsymbol{f}(\cdot)$ such that $\|\boldsymbol{f}\|_{L^{1}(0, T ; E)}=\int_{0}^{T}\|\boldsymbol{f}(t)\|_{E} d t<\infty$ endowed with $\|\cdot\|_{L^{1}(0, T: E)}$, where $\boldsymbol{E}$ is an arbitrary Banach space. If $\boldsymbol{E}^{*}$ is the strong dual of $\boldsymbol{E}$, an $\boldsymbol{E}^{*}$-valued function $\boldsymbol{\mu}(\cdot)$ is $\boldsymbol{E}$-weakly measurable if and only if

$$
<\boldsymbol{\mu}(\cdot), \boldsymbol{y}>_{E^{*} \times E} \quad \text { is measurable } \forall \boldsymbol{y} \in \boldsymbol{E} .
$$

The space of all such $\boldsymbol{\mu}(\cdot)$ with

$$
\left|<\boldsymbol{\mu}(t), \boldsymbol{y}>_{E^{*} \times E}\right| \leq C\|\boldsymbol{y}\|_{E}, \quad \text { a.e. in } 0 \leq t \leq T
$$

( the null set where (??) fails to hold will in general depend on $\boldsymbol{y}$ ) is named $L_{\mathrm{W}}^{\infty}\left(0, T ; \boldsymbol{E}^{*}\right)$ and equipped with the norm

$$
\|\boldsymbol{\mu}(\cdot)\|_{L_{\mathrm{W}}^{\infty}\left(0, T ; E^{*}\right)}=\text { infimum of all } C \text { such that }(? ?) \text { holds. }
$$

The equivalence relation in $L_{\mathrm{W}}^{\infty}\left(0, T ; \boldsymbol{E}^{*}\right)$ is

$$
\boldsymbol{\mu}(\cdot) \approx \boldsymbol{\nu}(\cdot) \text { if and only if }\|\boldsymbol{\mu}(\cdot)-\boldsymbol{\nu}(\cdot)\|_{L_{\mathrm{W}}^{\infty}\left(0, T ; E^{*}\right)}=0,
$$

that is, if

$$
<\boldsymbol{\mu}(t), \boldsymbol{y}>_{E^{*} \times E}=<\boldsymbol{\nu}(t), \boldsymbol{y}>_{E^{*} \times E} \quad \text { a.e. in } 0 \leq t \leq T .
$$

As before, the null set where the above relation fails to hold will depend on $\boldsymbol{y}$. A function $\boldsymbol{\mu}(\cdot) \in L_{\mathrm{W}}^{\infty}\left(0, T ; \boldsymbol{E}^{*}\right)$ produces an element in $\left(L^{1}(0, T ; \boldsymbol{E})\right)^{*}$ through

$$
<\boldsymbol{\mu}(\cdot), \boldsymbol{f}(\cdot)>=\int_{0}^{T}<\boldsymbol{\mu}(r), \boldsymbol{f}(r)>_{E^{*} \times E} d r, \quad \forall \boldsymbol{f}(\cdot) \in L^{1}(0, T ; \boldsymbol{E}) .(55)
$$

This functional only depends on the equivalence class of $\boldsymbol{\mu}(\cdot)$ and its norm coincides with the $L_{\mathrm{W}}^{\infty}\left(0, T ; \boldsymbol{E}^{*}\right)$-norm of $\boldsymbol{\mu}(\cdot)$. Conversely, it has been shown in $[?, ?]$ that every element of $\left(L^{1}(0, T ; \boldsymbol{E})\right)^{*}$ admits the representation (??) 
with $\boldsymbol{\mu}(\cdot) \in L_{\mathrm{W}}^{\infty}\left(0, T ; \boldsymbol{E}^{*}\right)$, thus $\left(L^{1}(0, T ; \boldsymbol{E})\right)^{*}=L_{\mathrm{W}}^{\infty}\left(0, T ; \boldsymbol{E}^{*}\right)$ algebraically and metrically. The proof is a consequence of the Dunford-Pettis theorem ([?], Theorem (6), page 503 and Lemma 8, page 504; [?], Corollary 1, page 89), which provides additional details: for instance, there exists a linear lifting operator $\boldsymbol{S}: L_{\mathrm{W}}^{\infty}\left(0, T ; \boldsymbol{E}^{*}\right) \rightarrow L_{\mathrm{W}}^{\infty}\left(0, T ; \boldsymbol{E}^{*}\right)$ such that $\boldsymbol{S} \boldsymbol{\mu}$ belongs to the equivalence class of $\boldsymbol{\mu}$ (in the sense defined earlier), $t \rightarrow\|[\boldsymbol{S} \boldsymbol{\mu}](t)\|_{E^{*}}$ is measurable in $0 \leq t \leq T$ and

$$
\sup _{0 \leq t \leq T}\|[\boldsymbol{S} \boldsymbol{\mu}](t)\|_{E^{*}}=\|\boldsymbol{\mu}(\cdot)\|_{L_{\mathrm{W}}^{\infty}\left(0, T ; E^{*}\right)} .
$$

Consider the Banach space $C_{\mathrm{b}}(\boldsymbol{U})$ of all bounded continuous functions in $\boldsymbol{U}$ equipped with the supremum norm, whose strong dual $C_{\mathrm{b}}(\boldsymbol{U})^{*}$ is isometrically isomorphic to the space $\boldsymbol{\Sigma}_{\mathrm{rba}}\left(\boldsymbol{U}, \boldsymbol{\Phi}_{\mathrm{C}}\right)$ of all bounded, finitely additive regular measures defined in the ring $\boldsymbol{\Phi}_{\mathrm{C}}$ generated by the closed sets of $\boldsymbol{U} ; \boldsymbol{\Sigma}_{\mathrm{rba}}\left(\boldsymbol{U}, \boldsymbol{\Phi}_{\mathrm{c}}\right)$ is equipped with the total variation norm ([?], page 258): $\|\mu\|_{\Sigma_{r b a}\left(U, \Phi_{c}\right)}:=|\mu|(\boldsymbol{U})$ with the total variation (which is also a finitely additive measure) defined as

$$
\forall A \in \boldsymbol{\Phi}_{\mathrm{C}}, \quad|\mu|(A)=\sup \sum\left|\mu\left(A_{j}\right)\right|
$$

where the supremum is taken over all finite sequences $\left\{A_{j}\right\}$ of disjoint sets in $\boldsymbol{\Phi}_{\mathrm{C}}$ with $A_{j} \subseteq A$. The duality pairing is

$$
<\boldsymbol{\mu}, f>_{C_{b}(U)^{*} \times C_{b}(U)}=\int_{\boldsymbol{U}} f(U) \mu(d U) .
$$

Here as noted above, the functional $\boldsymbol{\mu} \in C_{\mathrm{b}}(\boldsymbol{U})^{*}$ and the measure $\mu \in$ $\boldsymbol{\Sigma}_{\mathrm{rba}}\left(\boldsymbol{U}, \boldsymbol{\Phi}_{\mathrm{C}}\right)$ are isometrically and isomorphically identified.

Definition 1 Chattering Controls: The space $\mathcal{V}_{b c}(0, T ; \boldsymbol{U}, \kappa)$ of chattering controls consists of all

$$
\boldsymbol{\mu}(\cdot) \in\left(L^{1}\left(0, T ; C_{b}(\boldsymbol{U})\right)^{*}=L_{w}^{\infty}\left(0, T ; \boldsymbol{\Sigma}_{r b a}\left(\boldsymbol{U}, \boldsymbol{\Phi}_{c}\right)\right)\right.
$$

that satisfy

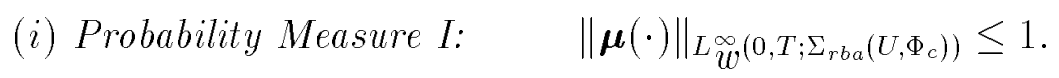

(ii) Probability Measure II: (Positive Cone Property) $\boldsymbol{f}(\cdot) \in L^{1}\left(0, T ; C_{b}(\boldsymbol{U})\right.$ ) is such that for $U \in \boldsymbol{U}, f(t, U) \geq 0$, a.e. in $0 \leq t \leq T$ then 


$$
\int_{0}^{T} \int_{\boldsymbol{U}} f(t, U) \mu(t, d U) d t \geq 0 .
$$

(iii) Probability Measure III: if $\mathcal{X} \boldsymbol{e}(\cdot)$ is the characteristic function of a measurable set $\boldsymbol{e} \subseteq[0, T]$ and $\mathcal{X}_{\boldsymbol{U}}(\cdot)$ is the function identically 1 in $\boldsymbol{U}$ then

$$
\begin{aligned}
& \int_{0}^{T} \int_{\boldsymbol{U}}\left(\mathcal{X}_{\boldsymbol{e}}(t) \otimes \mathcal{X}_{\boldsymbol{U}}(U)\right) \mu(t, d U) d t=\text { meas }(\boldsymbol{e}) . \\
& \text { (iv) Tightness Condition: } \quad \int_{\boldsymbol{U}} \kappa(U)^{2} \mu(\cdot, d U) \in L^{1}(0, T) .
\end{aligned}
$$

Remark 1 Note that ordinary controls (that is, controls in the space $\mathcal{U}_{\mathrm{ad}}(0, T ; \boldsymbol{U}, \kappa)$ defined in $\S 3$ ) can be duplicated by chattering controls. If $V(\cdot) \in \mathcal{U}_{\mathrm{ad}}(0, T ; \boldsymbol{U}, \kappa)$, we define a chattering control by the Dirac measure concentrated on $V(t)$ : $\boldsymbol{\mu}(t)=\boldsymbol{\delta}_{V(t)}$. It is obvious that $\boldsymbol{\mu}(t)$ satisfies conditions (i),(ii),(iii); as to (iv),

$$
\int_{\boldsymbol{U}} \kappa(U)^{2} \mu(\cdot, d U)=\kappa(V(\cdot))^{2}
$$

which belongs to $L^{1}(0, T)$ by the Control Space Hypothesis 1 .

Remark 2 Condition (iv) merits further explanation. Note that conditions (i), (ii) and (iii) are expressed entirely in terms of the action of $\boldsymbol{\mu}(\cdot)$ as a linear functional on $L^{1}\left(0, T ; C_{\mathrm{b}}(\boldsymbol{U})\right)$, so that they automatically respect the equivalence relation in $L_{\mathrm{W}}^{\infty}\left(0, T ; \boldsymbol{\Sigma}_{\mathrm{rba}}\left(\boldsymbol{U}, \boldsymbol{\Phi}_{\mathrm{C}}\right)\right)$. On the other hand, it is not clear that the integral in (iv), as a function of $t$, depends only on the equivalence class of $\boldsymbol{\mu}(\cdot)$; for that matter, it is not even clear that it is a measurable function of $t$. This is in fact so is shown below.

We use the theory of integration of scalar functions against finitely additive measures [?], of which we only need the particular case corresponding to bounded measures. A function $g(U)$ is $\boldsymbol{\mu}$-simple if $g(U)=\sum_{j=1}^{m} \alpha_{j} \mathcal{X}_{A_{j}}(U)$ for some $m<\infty$. Here $\mathcal{X}_{A_{j}}(\cdot)$ are the characteristic functions of pairwise disjoint sets $A_{j} \in \boldsymbol{\Phi}_{\mathrm{C}}$; by definition,

$$
\int_{\boldsymbol{U}} g(U) \mu(d U)=\sum_{j=1}^{m} \alpha_{j} \mu\left(A_{j}\right) .
$$

A function $f(U)$ defined in $\boldsymbol{U}$ is $\boldsymbol{\mu}$-measurable if and only if there exists a sequence $\left\{g_{k}(\cdot)\right\}$ of $\mu$-simple functions such that $g_{k}(\cdot) \rightarrow f(\cdot)$ in $|\mu|^{*}$-measure $\left(|\mu|^{*}\right.$ is the outer measure generated by the finitely additive measure $\left.|\mu|\right)$, that is $\forall \epsilon>0$, 


$$
\lim _{k \rightarrow \infty}|\mu|^{*}\left\{U \in \boldsymbol{U} ;\left|g_{k}(U)-f(U)\right| \geq \epsilon\right\}=0
$$

The class of $\mu$-measurable functions is closed under convergence in measure $([?]$, Lemma 11, page 106).

The function $f(U)$ is in addition $\boldsymbol{\mu}$-integrable if the sequence $\left\{g_{k}(\cdot)\right\}$ can be selected in such a way that

$$
\lim _{k, m \rightarrow \infty} \int_{\boldsymbol{U}}\left|g_{k}(U)-g_{m}(U)\right| \mu(d U)=0 .
$$

The integral is defined by

$$
\int_{\boldsymbol{U}} f(U) \mu(d U):=\lim _{k \rightarrow \infty} \int_{\boldsymbol{U}} g_{k}(U) \mu(d U)
$$

We shall only integrate functions in $C(\boldsymbol{U})$, the space of all (bounded and unbounded) continuous functions in $\boldsymbol{U}$. A function $f(\cdot) \in C(\boldsymbol{U})$ may not be $\mu$-measurable. If $f(U) \geq 0$ is not $\mu$-measurable, or if it is $\mu$-measurable but not $\mu$-integrable, we write (for a positive measure $\mu$ )

$$
\int_{\boldsymbol{U}} f(U) \mu(d U)=+\infty
$$

A key property of this integration theory is the

Proposition 3 Dominated Convergence Theorem: If $\left\{f_{n}(\cdot)\right\}$ is a sequence of $\mu$-measurable functions such that $f_{n}(\cdot) \rightarrow f(\cdot)$ in $|\mu|^{*}$-measure and such that $\left|f_{n}(U)\right| \leq g(U)$, where $g(\cdot)$ is $\mu$-integrable, then $f(\cdot)$ is $\mu$-integrable and

$$
\int_{\boldsymbol{U}} f(U) \mu(d U)=\lim _{n \rightarrow \infty} \int_{\boldsymbol{U}} f_{n}(U) \mu(d U) .
$$

For proofs and for additional facts see ([?], pages 109 and 124). We note that in its traditional formulation (a.e convergence) the dominated convergence theorem is false in the realm of finitely additive measures.

Lemma 3 Truncation Lemma: Let $f(\cdot) \in C(\boldsymbol{U}), f(U) \geq 0$. Then

$$
\int_{\boldsymbol{U}} f(U) \mu(d U)=\lim _{n \rightarrow \infty} \int_{\boldsymbol{U}} f(U)_{n} \mu(d U)
$$

where $f(U)_{n}=\min (f(U), n)$ and $\mu \in \boldsymbol{\Sigma}_{r b a}\left(\boldsymbol{U}, \boldsymbol{\Phi}_{c}\right)$ is a positive measure. 
Proof: We note that, since $\mu \in \boldsymbol{\Sigma}_{\mathrm{rba}}\left(\boldsymbol{U}, \boldsymbol{\Phi}_{\mathrm{c}}\right)=C_{\mathrm{b}}(\boldsymbol{U})^{*}$ and $f(U)_{n} \in$ $C_{\mathrm{b}}(\boldsymbol{U})$, the integrals (the duality pairings) on the right hand side of (??) always exists and are finite. On the other hand, the integral of $f(\cdot)$ may be $=+\infty$.

We begin by showing that if $f(U)$ is $\mu$-measurable then $f(U)_{n}$ converges to $f(U)$ in $\mu$-measure. Since

$$
\left\{U \in \boldsymbol{U} ; f(U)-f(U)_{n} \geq \epsilon\right\} \subseteq\{U \in \boldsymbol{U} ; f(U) \geq n\},
$$

it is enough to show that $\mu^{*}\{U \in \boldsymbol{U} ; f(U) \geq n\} \rightarrow 0$ as $n \rightarrow \infty$. Since $f(\cdot)$ is $\mu$-measurable, for a given $\delta$, we can find a $\mu$-simple function $g(U)$ such that $\mu^{*}\{U \in \boldsymbol{U} ;|f(U)-g(U)| \geq \epsilon\} \leq \delta$. Now, since $g(\cdot)$ is a $\mu$-simple function it is bounded and thus if $m>|\sup g(U)|$ we have

$$
\{U \in \boldsymbol{U} ; f(U) \geq m+1\} \subseteq\{U \in \boldsymbol{U} ;|f(U)-g(U)| \geq \epsilon\}
$$

Hence,

$$
\mu^{*}\{U \in \boldsymbol{U} ; f(U) \geq m+1\} \leq \mu^{*}\{U \in \boldsymbol{U} ;|f(U)-g(U)| \geq \epsilon\} \leq \delta,
$$

which establishes our claim.

Now, $f(\cdot)_{n} \rightarrow f(\cdot)$ in $\mu$-measure and $f(U)_{n} \leq f(U)$. Thus, if the integral $\int_{\boldsymbol{U}} f(U) \mu(d U)$ is finite, then (??) follows from the dominated convergence theorem.

On the other hand, assume that the integrals on the right hand side of (??) are uniformly bounded. Then, using the fact that $f(\cdot)$ and $\mu$ are positive

$$
\int_{\boldsymbol{U}} f(U)_{n} \mu(d U) \geq \int_{\{U \in \boldsymbol{U} ; f(U) \geq n\}} n \mu(d U)=n \mu\{U \in \boldsymbol{U} ; f(U) \geq n\}
$$

since $\{U \in \boldsymbol{U} ; f(U) \geq n\} \in \boldsymbol{\Phi}_{\mathrm{C}}$ due to the continuity of $f(\cdot)$. Thus, dividing this inequality by $n$ and taking the limit $n \rightarrow \infty$, we deduce that,

$$
\mu^{*}\{U \in \boldsymbol{U} ; f(U) \geq n\}=\mu\{U \in \boldsymbol{U} ; f(U) \geq n\} \rightarrow 0 \text { as } n \rightarrow \infty .
$$

Since $\left\{U \in \boldsymbol{U} ; f(U)-f(U)_{n} \geq \epsilon\right\} \subseteq\{U \in \boldsymbol{U} ; f(U) \geq n\}, f(U)_{n}$ converges to $f(U)$ in measure and thus $f(\cdot)$ is $\mu$-measurable. It remains to show that $f(\cdot)$ is $\mu$-integrable, in which case (??) holds due to previous considerations. We do this as follows. Let $\epsilon>0$. Since $f(U)_{n}$ is integrable, there exists a $\mu$-simple function $g_{n}(\cdot)$ such that 


$$
\mu^{*}\left\{U \in \boldsymbol{U} ;\left|f(U)_{n}-g_{n}(U)\right| \geq \epsilon / 2\right\} \leq 1 / n
$$

and

$$
\int_{\boldsymbol{U}}\left|f(U)_{n}-g_{n}(U)\right| \mu(d U) \leq 1 / n
$$

If $n>m$ we have

$$
\begin{aligned}
& \int_{\boldsymbol{U}}\left|g_{n}(U)-g_{m}(U)\right| \mu(d U) \\
& \leq 1 / n+1 / m+\int_{\boldsymbol{U}}\left|f(U)_{n}-f(U)_{m}\right| \mu(d U) \\
& =1 / n+1 / m+\int_{\boldsymbol{U}} f(U)_{n} \mu(d U)-\int_{\boldsymbol{U}} f(U)_{m} \mu(d U) \rightarrow 0,
\end{aligned}
$$

as $m, n \rightarrow \infty$. On the other hand,

$$
\begin{aligned}
& \left\{U \in \boldsymbol{U} ;\left|f(U)-g_{n}(U)\right| \geq \epsilon\right\} \\
& \subseteq\left\{U \in \boldsymbol{U} ;\left|f(U)_{n}-g_{n}(U)\right| \geq \epsilon / 2\right\} \cup\left\{U \in \boldsymbol{U} ; f(U)-f(U)_{n} \geq \epsilon / 2\right\}
\end{aligned}
$$

so that $g(U)_{n}$ converges to $f(U)$ in $\mu$-measure. In view of (??), $f(U)$ is $\mu$-integrable and by (??), (??) holds.

The following result shows that condition (iv) (equation (??)) in the definition ?? of chattering controls depends only on equivalence class of $\boldsymbol{\mu}(\cdot) \in$ $L_{\mathrm{W}}^{\infty}\left(0, T ; \boldsymbol{\Sigma}_{\mathrm{rba}}\left(\boldsymbol{U}, \boldsymbol{\Phi}_{\mathrm{C}}\right)\right)$.

Corollary 1 Let $f(\cdot) \in C(\boldsymbol{U})$ be a positive (not necessarily bounded) function and $\boldsymbol{\mu}(\cdot) \in L_{w}^{\infty}\left(0, T ; \boldsymbol{\Sigma}_{r b a}\left(\boldsymbol{U}, \boldsymbol{\Phi}_{c}\right)\right)$ be a positive measure. Let

$$
F(t)=\int_{\boldsymbol{U}} f(U) \mu(t, d U) \quad \text { and } F_{n}(t)=\int_{\boldsymbol{U}} f(U)_{n} \mu(t, d U) .
$$

Then

$$
F(t)=\lim _{n \rightarrow \infty} F_{n}(t), \quad 0 \leq t \leq T .
$$

In particular, $F(t)$ is measurable and does not change (except in a null set) if $\boldsymbol{\mu}(\cdot)$ is replaced by another element of its equivalence class in $L_{w}^{\infty}\left(0, T ; \boldsymbol{\Sigma}_{r b a}\left(\boldsymbol{U}_{,} \boldsymbol{\Phi}_{c}\right)\right)$. 
Proof: That (??) holds is immediate from the Truncation Lemma above. Due to the equivalence relation in $L_{\mathrm{W}}^{\infty}\left(0, T ; \boldsymbol{\Sigma}_{\mathrm{rba}}\left(\boldsymbol{U}, \boldsymbol{\Phi}_{\mathrm{C}}\right)\right), F_{n}(t)$ will not change (except in sets of measure zero) if $\boldsymbol{\mu}$ is replaced by another member of its equivalence class. To show that each $F_{n}(t)$ is measurable, note that $f(\cdot)_{n} \in C_{\mathrm{b}}(\boldsymbol{U})$ and thus the weak measurability of $\boldsymbol{\mu}(\cdot)$ provides the required result.

Remark 3 Conditions (i), (ii), (iii) in Definition ?? are not independent; in fact under (iii), conditions (i) and (ii) are equivalent. To see this assume first that (ii) and (iii) hold. To show that (i) holds it is enough to show that

$$
\int_{0}^{T}<\boldsymbol{\mu}(t), \boldsymbol{f}(t)>_{\Sigma_{r b a}\left(U ; \Phi_{c}\right) \times C_{b}(U)} d t \leq 1
$$

for every $\boldsymbol{f}(\cdot) \in L^{1}\left(0, T ; C_{\mathrm{b}}(\boldsymbol{U})\right)$ with $\|\boldsymbol{f}(\cdot)\|_{L^{1}\left(0, T ; C_{b}(U)\right)} \leq 1$, and we may assume that $\boldsymbol{f}(\cdot)$ is a countably valued function, since such functions are dense in $L^{1}\left(0, T ; C_{\mathrm{b}}(\boldsymbol{U})\right)$.

Now, let $f(\cdot) \in C_{\mathrm{b}}(\boldsymbol{U})$. Define $f_{+}(U)=\max (f(u), 0)$. Then $f_{+}(\cdot) \in$ $C_{\mathrm{b}}(\boldsymbol{U})$ and

$$
\left\|f_{+}\right\|_{C_{b}(U)} \leq\|f\|_{C_{b}(U)},\left\|f_{+}-g_{+}\right\|_{C_{b}(U)} \leq\|f-g\|_{C_{b}(U)}, \forall f(\cdot), g(\cdot) \in C_{\mathrm{b}}(\boldsymbol{U}) .
$$

The first estimate is obvious; the second follows from the inequality

$$
\left|f_{+}(U)-g_{+}(U)\right| \leq|f(U)-g(U)|, \forall U \in \boldsymbol{U} .
$$

This inequality is plain for $f(U), g(U) \geq 0$ or for $f(U), g(U)<0$. If $f(U) \geq 0$ and $g(U)<0$ then

$$
\left|f_{+}(U)-g_{+}(U)\right|=f(U), \quad|f(U)-g(U)|=f(U)-g(U) .
$$

The case $f(U)<0, g(U) \geq 0$ is treated in the same way. Finally, if we set $f^{-}(U)=(-f(U))^{+}$, we have $f=f^{+}-f^{-}$.

Let $\boldsymbol{f}(\cdot)$ be a countably valued function in $L^{1}\left(0, T ; C_{\mathrm{b}}(\boldsymbol{U})\right)$,

$$
\boldsymbol{f}(t)=f(t, U)=\sum \mathcal{X}_{\boldsymbol{e}_{j}}(t) f_{j}(U), \quad f_{j}(\cdot) \in C_{\mathrm{b}}(\boldsymbol{U}),
$$

$\mathcal{X}_{\boldsymbol{e}_{j}}(\cdot)$ the characteristic function of $\boldsymbol{e}_{j},\left\{\boldsymbol{e}_{j}\right\}$ a sequence of pairwise disjoint measurable subsets of $[0, T]$. The function $\boldsymbol{f}^{+}(t)=f^{+}(t, U)=\sum \mathcal{X}_{\boldsymbol{e}_{j}}(t) f_{j}^{+}(U)$ is countably valued and 


$$
\left\|\boldsymbol{f}^{+}(t)\right\|_{C_{b}(U)} \leq\|\boldsymbol{f}(t)\|_{C_{b}(U)}, \quad 0 \leq t \leq T,
$$

thus $\boldsymbol{f}^{+}(\cdot)$ belongs to $L^{1}\left(0, T ; C_{\mathrm{b}}(\boldsymbol{U})\right)$ as well with

$$
\left\|\boldsymbol{f}^{+}(\cdot)\right\|_{L^{1}\left(0, T ; C_{b}(U)\right)} \leq\|\boldsymbol{f}(\cdot)\|_{L^{1}\left(0, T ; C_{b}(U)\right)} \cdot
$$

Now, we have

$$
\begin{aligned}
& \int_{0}^{T}<\boldsymbol{\mu}(t), \boldsymbol{f}(t)>d t=\int_{0}^{T} \int_{\boldsymbol{U}} f(t, U) \mu(t, d U) d t \\
& =\int_{0}^{T} \int_{\boldsymbol{U}} f^{+}(t, U) \mu(t, d U) d t-\int_{0}^{T} \int_{\boldsymbol{U}} f^{-}(t, U) \mu(t, d U) d t \\
& \leq \sum \int_{0}^{T} \int_{\boldsymbol{U}} \mathcal{X}_{\boldsymbol{e}_{j}}(t) f_{j}^{+}(U) \mu(t, d U) d t \\
& \leq \sum \int_{0}^{T} \int_{\boldsymbol{U}}\left(\mathcal{X}_{\boldsymbol{e}_{j}}(t) \otimes \mathcal{X}_{\boldsymbol{U}}(U)\right)\left\|f_{j}^{+}\right\|_{C_{b}(U)} \mu(t, d U) d t \\
& =\sum \operatorname{meas}\left(\boldsymbol{e}_{j}\right)\left\|f_{j}^{+}\right\|_{C_{b}(U)}=\left\|\boldsymbol{f}^{+}(\cdot)\right\|_{L^{1}\left(0, T ; C_{b}(U)\right)} \leq\|\boldsymbol{f}(\cdot)\|_{L^{1}\left(0, T ; C_{b}(U)\right)}
\end{aligned}
$$

On the other hand, conditions (iii) and (i) imply (ii). This is a particular case of

Proposition 4 [?] Let $\boldsymbol{\nu}(\cdot) \in L_{\mathscr{W}}^{\infty}\left(0, T ; \boldsymbol{\Sigma}_{r b a}\left(\boldsymbol{U}, \boldsymbol{\Phi}_{c}\right)\right)$ satisfy properties (i) and (iii) of Definition ??. Then $\boldsymbol{\nu}(\cdot)$ satisfies (ii): precisely, there exists an element $\boldsymbol{\mu}(\cdot)$ in the equivalence class of $\boldsymbol{\nu}(\cdot)$ with

$$
\mu(t, A) \geq 0, \forall A \in \boldsymbol{\Phi}_{c} \text { and } \mu(t, \boldsymbol{U})=1 \text {, for } t \text { a.e. in } 0 \leq t \leq T \text {. }
$$

Proof: Let $\boldsymbol{S}$ be the lifting operator provided by the Dunford-Pettis theorem and define $\boldsymbol{\mu}(\cdot)=\boldsymbol{S} \boldsymbol{\nu}(\cdot)$. Since $\mu(t, \boldsymbol{U})=<\boldsymbol{\mu}(t), \mathcal{X}_{\boldsymbol{U}}(U)>$, the function $t \rightarrow \mu(t, \boldsymbol{U})$ is measurable. If $\mu(t, \boldsymbol{U})<1$ in a set of positive measure, we may find $\epsilon>0$ and a set $\boldsymbol{e}$ of positive measure such that

$$
\mu(t, \boldsymbol{U}) \leq 1-\epsilon, \quad t \in \boldsymbol{e} .
$$

If $\mathcal{X}_{\boldsymbol{e}}(\cdot)$ is the characteristic function of $\boldsymbol{e}$, then

$$
\int_{0}^{T} \int_{\boldsymbol{U}}\left(\mathcal{X}_{\boldsymbol{e}}(t) \otimes \mathcal{X}_{\boldsymbol{U}}(U)\right) \mu(t, d U) d t \leq \operatorname{meas}(\boldsymbol{e})(1-\epsilon)<\operatorname{meas}(\boldsymbol{e})
$$


which contradicts condition (iii) and shows the second equality in (??). Let $t$ belong to the set $\boldsymbol{d}$ where $\mu(t, \boldsymbol{U})=1$. Assume that there exists a set $A \subseteq \boldsymbol{U}$, $A \in \boldsymbol{\Phi}_{\mathrm{C}}$ such that $\mu(t, A)<0$. Then, denoting by $A^{c}$ the complement of $A$, $1=\mu(t, \boldsymbol{U})=\mu(t, A)+\mu\left(t, A^{c}\right)$, whereas

$$
\|\boldsymbol{\mu}(t)\|_{\Sigma_{r b a}\left(U ; \Phi_{c}\right)}=|\mu|(t, \boldsymbol{U}) \geq|\mu(t, A)|+\left|\mu\left(t, A^{c}\right)\right|>1 .
$$

This contradicts condition (i) and hence the first equality of (??) holds as well in $\boldsymbol{d}$.

Proposition ?? shows that in $\mathcal{V}_{\mathrm{bc}}(0, T ; \boldsymbol{U}, \kappa)$ every equivalence class of chattering control contains "nice" elements. Here we may even insure (??) to hold everywhere by redefining $\boldsymbol{\mu}(\cdot)$ as $\boldsymbol{\mu}(\cdot)=\boldsymbol{\delta}_{U}$ for some arbitrary $U \in \boldsymbol{U}$, for $t$ in the null set where (??) fails.

We proceed to the definition of the relaxed system. It will be of the form (??)-(??) in §3, but with different control set and control operator. The relaxed counterparts (of the control set $\boldsymbol{U}$ and control operator $\mathcal{N}$ ) will be denoted by $\Re \boldsymbol{U}$ and $\boldsymbol{N}$ respectively. isfy

Let $\boldsymbol{\Sigma}_{\text {rba }}\left(\boldsymbol{U}, \boldsymbol{\Phi}_{\mathrm{c}}, \kappa\right)$ be the subspace of $\boldsymbol{\Sigma}_{\text {rba }}\left(\boldsymbol{U}, \boldsymbol{\Phi}_{\mathrm{c}}\right)$ whose elements sat-

$$
\boldsymbol{\kappa}(\boldsymbol{\mu})^{2}:=\int_{\boldsymbol{U}} \kappa(U)^{2}|\mu|(d U)<\infty .
$$

The chattering (or relaxed) control set $\Re U$ corresponds to all $\mu \in$ $\boldsymbol{\Sigma}_{\text {rba }}\left(\boldsymbol{U}, \boldsymbol{\Phi}_{\mathrm{C}}, \kappa\right)$ that satisfy

$$
\mu(A) \geq 0, \forall A \in \boldsymbol{\Phi}_{\mathrm{C}} \text { and } \mu(\boldsymbol{U})=1 .
$$

Although $\boldsymbol{\Sigma}_{\mathrm{rba}}\left(\boldsymbol{U}, \boldsymbol{\Phi}_{\mathrm{C}}, \kappa\right)$, equipped with $\|\cdot\|_{\kappa}:=\boldsymbol{\kappa}(\cdot)^{2}$ is a Banach space, this is not essential here since no structure was required on the control set $\boldsymbol{U}$ in $§ 3$. In view of Proposition ?? (and modulo equivalence) our chattering controls take values in $\Re \boldsymbol{U}$ and satisfy the Control Space Hypothesis 1 (in §3) with $\boldsymbol{\kappa}(\boldsymbol{\mu})$ playing the part of $\kappa(U)$.

The relaxed control operator $\boldsymbol{N}: \boldsymbol{X}_{1 / 2} \times \Re \boldsymbol{U} \rightarrow \boldsymbol{X}$ will be denoted $\boldsymbol{N}(\boldsymbol{v}) \boldsymbol{\mu}$ to emphasize the linearity in $\boldsymbol{\mu}$ and is defined in the following way: $\boldsymbol{N}(\boldsymbol{v}) \boldsymbol{\mu}$ is the unique element of $\boldsymbol{X}$ satisfying 


$$
(\boldsymbol{z}, \boldsymbol{N}(\boldsymbol{v}) \boldsymbol{\mu})=\int_{\boldsymbol{U}}(\boldsymbol{z}, \mathcal{N}(\boldsymbol{v}, U)) \mu(d U), \quad \forall \boldsymbol{z} \in \boldsymbol{X}
$$

In view of Theorem ?? the integral exists. In fact, using the Schwarz inequality, estimate (??) and the fact that $\mu(\boldsymbol{U})=1$,

$$
\begin{aligned}
& |(\boldsymbol{z}, \boldsymbol{N}(\boldsymbol{v}) \boldsymbol{\mu})| \leq\|\boldsymbol{z}\| \int_{\boldsymbol{U}}\|\mathcal{N}(\boldsymbol{v}, U)\| \mu(d U) \leq\|\boldsymbol{z}\| \int_{\boldsymbol{U}} K_{N} \kappa(U) \mu(d U) \\
& \leq K_{N}\|\boldsymbol{z}\|\left\{\int_{\boldsymbol{U}} \mu(d U)\right\}^{1 / 2}\left\{\int_{\boldsymbol{U}} \kappa(U)^{2} \mu(d U)\right\}^{1 / 2} .
\end{aligned}
$$

Thus, the linear functional

$$
\boldsymbol{z} \rightarrow \int_{\boldsymbol{U}}(\boldsymbol{z}, \mathcal{N}(\boldsymbol{v}, U)) \mu(d U)
$$

is continuous from $\boldsymbol{X} \rightarrow \boldsymbol{R}$ and by the Riesz representation theorem, there exists a unique element $\boldsymbol{N}(\boldsymbol{v}) \boldsymbol{\mu}$ in $\boldsymbol{X}$ which satisfies

$$
\|\boldsymbol{N}(\boldsymbol{v}) \boldsymbol{\mu}\| \leq K_{N} \boldsymbol{\kappa}(\boldsymbol{\mu}), \text { for } \boldsymbol{v} \in \boldsymbol{X}_{1 / 2},\|\boldsymbol{v}\|_{1 / 2} \leq C
$$

This is similar to inequality (??) with the function $\boldsymbol{\kappa}(\boldsymbol{\mu})$ playing the role of $\kappa(U)$. A result similar to the continuity estimate (??) in Theorem ?? can be obtained from

Lemma $4 \boldsymbol{N}(\boldsymbol{v}) \boldsymbol{\mu}$ is continuous in $\boldsymbol{X}_{1 / 2} \times \Re \boldsymbol{U}$ and locally Lipschitz continuous: if $L_{N}=L_{N}(C)$ is the constant in (??) then

$$
\begin{aligned}
& \|\boldsymbol{N}(\boldsymbol{v}) \boldsymbol{\mu}-\boldsymbol{N}(\boldsymbol{z}) \boldsymbol{\mu}\| \leq L_{N}\|\boldsymbol{v}-\boldsymbol{z}\|_{1 / 2} \boldsymbol{\kappa}(\boldsymbol{\mu}), \\
& \text { for } \boldsymbol{v} \in \boldsymbol{X}_{1 / 2}, \boldsymbol{z} \in \boldsymbol{X}_{1 / 2}, \boldsymbol{\mu} \in \Re \boldsymbol{U} \text { and }\|\boldsymbol{v}\|_{1 / 2},\|\boldsymbol{z}\|_{1 / 2} \leq C .
\end{aligned}
$$

Proof: We have

$$
\boldsymbol{N}(\boldsymbol{v}) \boldsymbol{\nu}-\boldsymbol{N}(\boldsymbol{z}) \boldsymbol{\mu}=(\boldsymbol{N}(\boldsymbol{v}) \boldsymbol{\nu}-\boldsymbol{N}(\boldsymbol{v}) \boldsymbol{\mu})+(\boldsymbol{N}(\boldsymbol{v}) \boldsymbol{\mu}-\boldsymbol{N}(\boldsymbol{z}) \boldsymbol{\mu}) .
$$

For the first parenthesis we use (??). For the second using estimate (??),

$$
\begin{aligned}
& |(\boldsymbol{w}, \boldsymbol{N}(\boldsymbol{v}) \boldsymbol{\mu}-\boldsymbol{N}(\boldsymbol{z}) \boldsymbol{\mu})| \leq \int_{\boldsymbol{U}}|(\boldsymbol{w}, \mathcal{N}(\boldsymbol{v}, U)-\mathcal{N}(\boldsymbol{z}, U))| \mu(d U) \\
& \leq L_{N}\|\boldsymbol{w}\| \int_{\boldsymbol{U}} \kappa(U)\|\boldsymbol{v}-\boldsymbol{z}\|_{1 / 2} \mu(d U) .
\end{aligned}
$$


Applying the Schwarz inequality

$$
\begin{aligned}
& |(\boldsymbol{w}, \boldsymbol{N}(\boldsymbol{v}) \boldsymbol{\mu}-\boldsymbol{N}(\boldsymbol{z}) \boldsymbol{\mu})| \\
& \leq L_{N}\|\boldsymbol{w}\|\|\boldsymbol{v}-\boldsymbol{z}\|_{1 / 2}\left\{\int_{\boldsymbol{U}} \mu(d U)\right\}^{1 / 2}\left\{\int_{\boldsymbol{U}} \kappa(U)^{2} \mu(d U)\right\}^{1 / 2} .
\end{aligned}
$$

This gives the required estimate (??).

To apply the existence results in $§ 3$, it only remains to verify the strong measurability of the control operator $\boldsymbol{N}(\boldsymbol{v}(\cdot)) \boldsymbol{\mu}(\cdot)$. This is given by

Lemma 5 Let $\boldsymbol{v}(\cdot) \in C\left(0, T ; \boldsymbol{X}_{1 / 2}\right), \boldsymbol{\mu}(\cdot) \in \mathcal{V}_{b c}(0, T ; \boldsymbol{U}, \kappa)$. Then $\boldsymbol{N}(\boldsymbol{v}(\cdot)) \boldsymbol{\mu}(\cdot)$ is strongly measurable.

Proof: The space $\boldsymbol{X}$ is separable, thus it is enough to show weak measurability of $\boldsymbol{N}(\boldsymbol{v}(\cdot)) \boldsymbol{\mu}(\cdot)$, that is, measurability of

$$
\begin{aligned}
& t \rightarrow(\boldsymbol{z}, \boldsymbol{N}(\boldsymbol{v}(t)) \boldsymbol{\mu}(t))=\int_{\boldsymbol{U}}(\boldsymbol{z}, \mathcal{N}(\boldsymbol{v}(t), U)) \mu(t, d U) \\
& =\int_{\boldsymbol{U}} \kappa(U)\left\{\kappa(U)^{-1}(\boldsymbol{z}, \mathcal{N}(\boldsymbol{v}(t), U))\right\} \mu(t, d U)
\end{aligned}
$$

for $\boldsymbol{z} \in \boldsymbol{X}$ arbitrary. For each $t$, define a (finitely additive) measure $\boldsymbol{\lambda}(t)$ by

$$
\lambda(t, A)=\int_{A} \kappa(U)^{-1}(\boldsymbol{z}, \mathcal{N}(\boldsymbol{v}(t), U)) \mu(t, d U), \quad A \in \boldsymbol{\Phi}_{\mathrm{C}}
$$

Then, $|\lambda|(t, \cdot)$ is given by

$$
|\lambda|(t, A)=\int_{A} \kappa(U)^{-1}|(\boldsymbol{z}, \mathcal{N}(\boldsymbol{v}(t), U))| \mu(t, d U),
$$

so that

$$
\|\lambda(t)\|_{\Sigma_{r b a}\left(U, \phi_{c}\right)}=|\lambda|(t, \boldsymbol{U}) \leq K_{N}\|\boldsymbol{z}\| \mu(t, \boldsymbol{U})=K_{N}\|\boldsymbol{z}\|
$$

and $\boldsymbol{\lambda}(t) \in \boldsymbol{\Sigma}_{\mathrm{rba}}\left(\boldsymbol{U}, \boldsymbol{\Phi}_{\mathrm{c}}\right)$. For $f(\cdot) \in C_{\mathrm{b}}(\boldsymbol{U})$ we have

$$
\int_{\boldsymbol{U}} f(U) \lambda(t, d U)=\int_{\boldsymbol{U}} f(U)\left\{\kappa(U)^{-1}(\boldsymbol{z}, \mathcal{N}(\boldsymbol{v}(t), U))\right\} \mu(t, d U)
$$


By Theorem ??, the function $t \rightarrow f(\cdot)\left\{\kappa(\cdot)^{-1}(\boldsymbol{z}, \mathcal{N}(\boldsymbol{v}(t), \cdot))\right\}$ is continuous from $[0, T]$ into $C_{\mathrm{b}}(\boldsymbol{U})$, thus a foriori it belongs to $L^{1}\left(0, T ; C_{\mathrm{b}}(\boldsymbol{U})\right)$ and it follows that (??) is a measurable function of $t$, thus $\boldsymbol{\lambda}(\cdot) \in L_{\mathrm{W}}^{\infty}\left(0, T ; \boldsymbol{\Sigma}_{\mathrm{rba}}\left(\boldsymbol{U}, \boldsymbol{\Phi}_{\mathrm{C}}\right)\right)$. A similar argument replacing $(\boldsymbol{z}, \mathcal{N}(\boldsymbol{v}(t), \cdot))$ by $|(\boldsymbol{z}, \mathcal{N}(\boldsymbol{v}(t), \cdot))|$ shows that $|\boldsymbol{\lambda}|(\cdot) \in L_{\mathrm{W}}^{\infty}\left(0, T ; \boldsymbol{\Sigma}_{\mathrm{rba}}\left(\boldsymbol{U}, \boldsymbol{\Phi}_{\mathrm{c}}\right)\right)$. Now, by virtue of Corollary ??,

$$
\int_{\boldsymbol{U}} \kappa(U)|\lambda|(t, d U)=\lim _{n \rightarrow \infty} \int_{\boldsymbol{U}} \kappa(U)_{n}|\lambda|(t, d U)
$$

On the other hand, if $m>n$,

$$
\left|\int_{\boldsymbol{U}} \kappa(U)_{m} \lambda(t, d U)-\int_{\boldsymbol{U}} \kappa(U)_{n} \lambda(t, d U)\right| \leq \int_{\boldsymbol{U}}\left(\kappa(U)_{m}-\kappa(U)_{n}\right)|\lambda|(t, d U)
$$

so that a relation similar to (??) holds as well for $\boldsymbol{\lambda}(\cdot)$. Accordingly, the function $t \rightarrow(\boldsymbol{z}, \boldsymbol{N}(\boldsymbol{v}(t)) \boldsymbol{\mu}(t))$ is the almost everywhere limit of

$$
t \rightarrow \int_{\boldsymbol{U}} \kappa(U)_{n}\left\{\kappa(U)^{-1}(\boldsymbol{z}, \mathcal{N}(\boldsymbol{v}(t), U))\right\} \mu(t, d U)
$$

which is measurable since the function $t \rightarrow \kappa(\cdot)_{n}\left\{\kappa(\cdot)^{-1}(\boldsymbol{z}, \mathcal{N}(\boldsymbol{v}(t), \cdot))\right\}$ is continuous from $[0, T]$ into $C_{\mathrm{b}}(\boldsymbol{U})$, thus it belongs to $L^{1}\left(0, T ; C_{\mathrm{b}}(\boldsymbol{U})\right)$.

The relaxed system corresponding to $\mathcal{V}_{\mathrm{bc}}(0, T ; \boldsymbol{U}, \kappa)$ is

$$
\boldsymbol{v}_{t}(t)+\mathcal{A} \boldsymbol{v}(t)+\mathcal{B}(\boldsymbol{v}(t))=\boldsymbol{N}(\boldsymbol{v}(t)) \boldsymbol{\mu}(t)
$$

and the relaxed cost functional is

$$
\boldsymbol{C}(t, \boldsymbol{\mu})=\int_{0}^{t} \boldsymbol{\Theta}(\boldsymbol{v}(r, \boldsymbol{\mu})) \boldsymbol{\mu}(r) d r
$$

where

$$
\boldsymbol{\Theta}(\boldsymbol{v}) \boldsymbol{\mu}=\int_{\boldsymbol{U}} \Theta(\boldsymbol{v}, U) \mu(d U), \text { for } \boldsymbol{\mu} \in \Re \boldsymbol{U}
$$

Note that by virtue of (??) we have

$$
|\boldsymbol{\Theta}(\boldsymbol{v}) \boldsymbol{\mu}| \leq K_{\Theta} \boldsymbol{\kappa}(\boldsymbol{\mu})^{2}, \quad \boldsymbol{v} \in \boldsymbol{X}_{1 / 2}, \boldsymbol{\mu} \in \Re \boldsymbol{U} \text { and }\|\boldsymbol{v}\|_{1 / 2} \leq C
$$

Lemma $6 \Theta(\boldsymbol{v}) \boldsymbol{\mu}$ is continuous in $\boldsymbol{X}_{1 / 2} \times \Re \boldsymbol{U}$. 
Lemma 7 Let $\boldsymbol{v}(\cdot) \in C\left(0, T ; \boldsymbol{X}_{1 / 2}\right), \boldsymbol{\mu}(\cdot) \in \mathcal{V}_{b c}(0, T ; \boldsymbol{U}, \kappa)$. Then $\boldsymbol{\Theta}(\boldsymbol{v}(\cdot)) \boldsymbol{\mu}(\cdot)$ is measurable.

Proofs follow the lines of that of Lemma?? and Lemma ?? and are omitted. Obviously, Lemma ?? implies the Control Space Hypothesis 2 for $\mathcal{V}_{\mathrm{bc}}(0, T ; \boldsymbol{U}, \kappa)$.

We have proved at this point that the relaxed system (??) satisfies all hypotheses necessary to apply the existence theory (Lemma?? and Lemma ??).

Remark We note an important special case of the theory. We noted in $\$ 2$ that when the spatial distribution of the boundary control is prescribed, the control set $\boldsymbol{U}$ can be taken as a closed subset of $\boldsymbol{R}^{m}$. If this set is bounded (in this case $\boldsymbol{U}$ would be compact) then by Alexandrov's theorem ([?], page 136.) each measure $\boldsymbol{\mu} \in \boldsymbol{\Sigma}_{\mathrm{rba}}\left(\boldsymbol{U}, \boldsymbol{\Phi}_{\mathrm{C}}\right)$ is actually $\sigma$-additive and admits a unique, regular countably additive extension to the $\sigma$-ring $\boldsymbol{\Phi}_{\mathrm{b}}$ generated by the closed sets of $\boldsymbol{U}$; in other words, each $\boldsymbol{\mu}$ can be extended to a bounded Borel measure in the Borel ring $\boldsymbol{\Phi}_{\mathrm{b}}$. Thus, in this case, the instantaneous values of our chattering controls are bona fide probability measures.

We cover finally the second (and enormously general) definition of chattering controls. In it, nothing is assumed of $\boldsymbol{U}$. The basic space is the Banach space $\boldsymbol{B}(\boldsymbol{U})$ of all bounded functions in $\boldsymbol{U}$ equipped with the supremum norm, whose dual $\boldsymbol{B}(\boldsymbol{U})^{*}$ is isometrically isomorphic to the space $\boldsymbol{\Sigma}_{\mathrm{ba}}(\boldsymbol{U}, \boldsymbol{\Phi})$ of all bounded, finitely additive measures defined in the ring $\boldsymbol{\Phi}$ of all subsets of $\boldsymbol{U} ; \boldsymbol{\Sigma}_{\mathrm{ba}}(\boldsymbol{U}, \boldsymbol{\Phi})$ is equipped with the total variation norm ([?], page 258.) and the duality pairing between $\boldsymbol{B}(\boldsymbol{U})$ and $\boldsymbol{\Sigma}_{\mathrm{ba}}(\boldsymbol{U}, \boldsymbol{\Phi})$ is again (??). The space $\mathcal{V}_{\mathrm{b}}(0, T ; \boldsymbol{U}, \kappa)$ of chattering controls consists of all

$$
\boldsymbol{\mu}(\cdot) \in L^{1}(0, T ; \boldsymbol{B}(\boldsymbol{U}))^{*}=L_{\mathrm{W}}^{\infty}\left(0, T ; \boldsymbol{\Sigma}_{\mathrm{ba}}(\boldsymbol{U}, \boldsymbol{\Phi})\right)
$$

that satisfy properties (i), (ii), (iii) and (iv) of Definition ??. In this case we modify Control Space Hypothesis 2 as

Control Space Hypothesis 3 For every $\boldsymbol{v}(\cdot) \in C\left([0, T] ; \boldsymbol{X}_{1 / 2}\right)$ and every $U(\cdot) \in \mathcal{U}_{a d}(0, T ; \boldsymbol{U}, \kappa), \Theta(\boldsymbol{v}(\cdot), U(\cdot))$ is measurable. Moreover, $\kappa(U)^{-2} \Theta(\boldsymbol{v}, U)$ is continuous with respect to $\boldsymbol{v}$ in $\boldsymbol{X}_{1 / 2} \times \boldsymbol{U}$, uniformly for $\boldsymbol{v}$ bounded and $U \in \boldsymbol{U}$.

The uniform continuity stated above can also be expressed as 


$$
\sup \left\{\left|\kappa(U)^{-2} \Theta(\boldsymbol{w}, U)-\kappa(U)^{-2} \Theta(\boldsymbol{v}, U)\right| ; U \in \boldsymbol{U}\right\} \rightarrow 0
$$

as $\|\boldsymbol{w}-\boldsymbol{v}\|_{1 / 2} \rightarrow 0$ uniformly for $\boldsymbol{w}, \boldsymbol{v}$ belong to a bounded set in $\boldsymbol{X}_{1 / 2}$.

\section{Existence results for the optimal problem}

We denote by $\mathcal{C}_{m}$ the infimum of the cost functional $\mathcal{C}(t, U)$ taken over all $U(\cdot) \in \mathcal{U}_{\mathrm{ad}}(0, t ; \boldsymbol{U}, \kappa)$ such that the trajectory $\boldsymbol{v}(t, U)$ satisfies the target condition

$$
\boldsymbol{v}(t, U) \in \boldsymbol{Y}
$$

We define the relaxed infimum $\boldsymbol{C}_{m}$ in the same way in relation to $\mathcal{V}_{\mathrm{bc}}(0, t ; \boldsymbol{U}, \kappa)$.

Since there are more relaxed controls than ordinary controls, we have $\boldsymbol{C}_{m} \leq \mathcal{C}_{m}$. We assume that

$$
-\infty<\boldsymbol{C}_{m}, \mathcal{C}_{m}<\infty
$$

The first inequality states that we cannot reach the target set with relaxed trajectories yielding arbitrarily low values of the cost functional, which would preclude existence of solutions of the optimal control problem. The second expresses that we can hit the target set with some ordinary trajectory, which is a precondition for the formulation of the optimal control problem. A minimizing sequence of ordinary controls is a sequence $\left\{U_{n}(\cdot)\right\}, U^{n} \in$ $\mathcal{U}_{\text {ad }}\left(0, t_{n} ; \boldsymbol{U}, \kappa\right)$ such that

$$
\limsup _{n \rightarrow \infty} \mathcal{C}\left(t_{n}, U^{n}\right) \leq \mathcal{C}_{m} \quad \text { and } \quad \lim _{n \rightarrow \infty} \operatorname{dist}\left(\boldsymbol{v}\left(t_{n}, U^{n}\right), \boldsymbol{Y}\right) \rightarrow 0 .
$$

Here the distance is in $\boldsymbol{X}$.

Minimizing sequences $\left\{\boldsymbol{\mu}_{n}(\cdot)\right\}, \boldsymbol{\mu}_{n}(\cdot) \in \mathcal{V}_{\mathrm{bc}}\left(0, t_{n} ; \boldsymbol{U}, \kappa\right)$ of chattering controls are defined in the same fashion in relation to the relaxed minimum $\boldsymbol{C}_{m}$ :

$$
\limsup _{n \rightarrow \infty} \boldsymbol{C}\left(t_{n}, \boldsymbol{\mu}_{n}\right) \leq \boldsymbol{C}_{m}, \quad \text { and } \lim _{n \rightarrow \infty} \operatorname{dist}\left(\boldsymbol{v}\left(t_{n}, \boldsymbol{\mu}_{n}\right), \boldsymbol{Y}\right) \rightarrow 0 .
$$

Again the distance is in $\boldsymbol{X}$.

Lemma 8 Let $\left\{\boldsymbol{\mu}_{\alpha}(\cdot)\right\}$ be a generalized sequence of chattering controls in $\mathcal{V}_{b c}(0, T ; \boldsymbol{U}, \kappa)$ such that 


$$
\boldsymbol{\kappa}\left(\boldsymbol{\mu}_{\alpha}(\cdot)\right)^{2}=\int_{\boldsymbol{U}} \kappa(U)^{2} \mu_{\alpha}(\cdot, d U)
$$

is uniformly bounded in $L^{1}(0, T)$. Then there exists a generalized subsequence, denoted also $\left\{\boldsymbol{\mu}_{\alpha}(\cdot)\right\}$, such that

$$
\boldsymbol{\mu}_{\alpha}(\cdot) \rightarrow \boldsymbol{\mu}(\cdot) \in \mathcal{V}_{b c}(0, T ; \boldsymbol{U}, \kappa)
$$

in the $L^{1}\left(0, T ; C_{b}(\boldsymbol{U})\right)$-weak topology and

$$
\left\|\boldsymbol{\kappa}(\boldsymbol{\mu}(\cdot))^{2}\right\|_{L^{1}(0, T)} \leq \liminf \left\|\boldsymbol{\kappa}\left(\boldsymbol{\mu}_{\alpha}(\cdot)\right)^{2}\right\|_{L^{1}(0, T)} .
$$

Proof: The space $\mathcal{V}_{\mathrm{bc}}(0, T ; \boldsymbol{U}, \kappa)$ is a subspace of the unit ball of the Banach space $L_{\mathrm{W}}^{\infty}\left(0, T ; \boldsymbol{\Sigma}_{\mathrm{rba}}(\boldsymbol{U}, \boldsymbol{\Phi})\right)=\left(L^{1}\left(0, T ; C_{\mathrm{b}}(\boldsymbol{U})\right)\right)^{*}$, thus by the BanachAlaoglu theorem, a $L^{1}\left(0, T ; C_{\mathrm{b}}(\boldsymbol{U})\right)$-weakly convergent generalized subsequence $\left\{\boldsymbol{\mu}_{\alpha}(\cdot)\right\}$ exists. Accordingly, we only have to check that the weak limit $\boldsymbol{\mu}(\cdot)$ satisfies (i),(ii), (iii) and (iv) of Definition ??. This is obvious for (i), (ii) and (iii). We will now verify (iv). If we have

$$
\left\|\int_{\boldsymbol{U}} \kappa(U)^{2} \mu_{\alpha}(\cdot, d U)\right\|_{L^{1}(0, T)} \leq C
$$

then, since $\boldsymbol{\mu}_{\alpha}(t, \cdot) \geq 0$ and $\left(\kappa(U)^{2}\right)_{n} \leq \kappa(U)^{2}$,

$$
\left\|\int_{\boldsymbol{U}}\left(\kappa(U)^{2}\right)_{n} \mu_{\alpha}(\cdot, d U)\right\|_{L^{1}(0, T)} \leq C .
$$

Since the constant function $t \rightarrow\left(\kappa(U)^{2}\right)_{n}$ belongs to $L^{1}\left(0, T ; C_{\mathrm{b}}(\boldsymbol{U})\right)$, we deduce that

$$
\int_{0}^{T} \int_{\boldsymbol{U}}\left(\kappa(U)^{2}\right)_{n} \mu_{\alpha}(t, d U) d t \rightarrow \int_{0}^{T} \int_{\boldsymbol{U}}\left(\kappa(U)^{2}\right)_{n} \mu(t, d U) d t .
$$

Taking into account of the fact that the measures are positive, (??) expresses convergence of the $L^{1}$ norms: in view of (??),

$$
\left\|\int_{U}\left(\kappa(U)^{2}\right)_{n} \mu(\cdot, d U)\right\|_{L^{1}(0, T)} \leq C .
$$

Now, using Corollary ?? of the truncation lemma and Fatou's theorem, the results follows. 
Our next convergence result (Lemma 9) uses the dual of $L^{2}\left(0, T ; C_{\mathrm{b}}(\boldsymbol{U})\right)$. The dual of $L^{p}(0, T ; \boldsymbol{E})$ for $1<p<\infty$ and $\boldsymbol{E}$ an arbitrary Banach space has been characterized in ([?], page 97$)$. Let us recall below certain relevant aspects of this theory.

Let $f(t)$ be a nonnegative, possibly nonmeasurable function in the interval $0 \leq t \leq T$. The upper integral ${ }^{*} \int f(t) d t$ is defined by

$$
* \int_{0}^{T} f(t) d t=\inf \int_{0}^{T} g(t) d t
$$

the infimum taken over all measurable functions with $g(t) \geq f(t)$; if for all such $g$ we have $\int g(t) d t=\infty$, we define ${ }^{*} \int f(t) d t=+\infty$. Obviously, ${ }^{*} \int f(t) d t=\int f(t) d t$ if $f(\cdot)$ is integrable; also, ${ }^{*} \int \lambda f(t) d t=\lambda\left({ }^{*} \int f(t) d t\right)$ for $\lambda \geq 0$ and

$$
* \int(f(t)+g(t)) d t \leq^{*} \int f(t) d t+{ }^{*} \int g(t) d t \text { for arbitrary } f, g .
$$

We note also that if ${ }^{*} \int f(t) d t=0$, then there exists a sequence $\left\{g_{n}(\cdot)\right\}$ of integrable nonnegative functions such that $g_{n}(t) \geq f(t)$ and $\int g_{n}(t) d t \rightarrow 0$. Replacing the sequence by $g_{1}, \min \left(g_{1}, g_{2}\right), \min \left(g_{1}, g_{2}, g_{3}\right), \cdots$ we may assume that it is decreasing, hence pointwise convergent to a nonnegative integrable function $g(t)$ whose integral, by Fatou's theorem has to be zero. It then follows that $f(t)=0$ a.e.

We consider below $\boldsymbol{E}^{*}$-valued functions $\boldsymbol{\mu}(\cdot)$ which are weakly measurable in the sense of $\$ 4$, with the same equivalence relation. The space $L_{\mathrm{W}}^{q}\left(0, T ; \boldsymbol{E}^{*}\right)$ consists of (equivalence classes of ) $\boldsymbol{E}$-weakly measurable $\boldsymbol{E}^{*}$ valued functions such that the equivalence class $[\boldsymbol{\mu}(\cdot)]$ of $\boldsymbol{\mu}(\cdot)$ contains some element $\boldsymbol{\nu}(\cdot)$ with ${ }^{*} \int\|\boldsymbol{\nu}(t)\|_{E^{*}}^{q} d t<\infty$. The norm in the space $L_{\mathrm{W}}^{q}\left(0, T ; \boldsymbol{E}^{*}\right)$ is defined by

$$
\|[\boldsymbol{\mu}(\cdot)]\|_{L_{\mathrm{W}}^{q}\left(0, T ; E^{*}\right)}=\inf _{\boldsymbol{\nu} \in[\boldsymbol{\mu}]}\left(* \int_{0}^{T}\|\boldsymbol{\nu}\|_{E^{*}}^{q} d t\right)^{1 / q} .
$$

It is obvious from the properties of ${ }^{*} \int$ that $\|[\boldsymbol{\mu}(\cdot)]\|_{L_{\mathrm{W}}^{q}\left(0, T ; E^{*}\right)}$ satisfies the properties of a norm. The space $L_{\mathrm{W}}^{q}\left(0, T ; \boldsymbol{E}^{*}\right)$ can be identified algebraically and metrically with the dual $L^{p}(0, T ; \boldsymbol{E})^{*}, 1 / p+1 / q=1$. The canonical pairing is again given by (??)

$$
<\boldsymbol{\mu}(\cdot), \boldsymbol{f}(\cdot)>=\int_{0}^{T}<\boldsymbol{\mu}(r), \boldsymbol{f}(r)>_{E^{*} \times E} d r, \quad \forall \boldsymbol{f}(\cdot) \in L^{p}(0, T ; \boldsymbol{E}) .(85)
$$


The following result complements Lemma ??.

Lemma 9 Let $\left\{\boldsymbol{\mu}_{\alpha}(\cdot)\right\}$ be the $L^{1}\left(0, T ; C_{b}(\boldsymbol{U})\right)$-weakly convergent generalized sequence in Lemma ??, $\boldsymbol{\mu}(\cdot)$ its limit. Then there exists a generalized subsequence (also denoted $\left\{\boldsymbol{\mu}_{\alpha}(\cdot)\right\}$ ) such that

$$
\int_{0}^{T} \int_{\boldsymbol{U}} f(t, U) \mu_{\alpha}(t, d U) d t \rightarrow \int_{0}^{T} \int_{\boldsymbol{U}} f(t, U) \mu(t, d U) d t
$$

for all $\boldsymbol{f}(t, U)$ such that $t \rightarrow \kappa(\cdot)^{-1} \boldsymbol{f}(t, \cdot)$ is in $L^{2}\left(0, T ; C_{b}(\boldsymbol{U})\right)$.

Proof: Consider the measure defined by

$$
\lambda_{\alpha}(t, A)=\int_{A} \kappa(U) \mu_{\alpha}(t, d U), \text { for } A \in \boldsymbol{\Phi}_{\mathrm{C}} .
$$

(informally, $\lambda_{\alpha}(t, d U)=\kappa(U) \mu_{\alpha}(t, d U)$ ). Then $\lambda_{\alpha}(t, \cdot)$ is a positive, finitely additive measure in $\boldsymbol{\Phi}_{\mathrm{c}}$. We have

$$
\begin{aligned}
& \left\|\boldsymbol{\lambda}_{\alpha}(t)\right\|_{\Sigma_{r b a}\left(U, \Phi_{c}\right)}=\int_{\boldsymbol{U}} \lambda_{\alpha}(t, d U)=\int_{\boldsymbol{U}} \kappa(U) \mu_{\alpha}(t, d U) \\
& \leq\left(\int_{\boldsymbol{U}} \kappa(U)^{2} \mu_{\alpha}(t, d U)\right)^{1 / 2}\left(\int_{\boldsymbol{U}} \mu_{\alpha}(t, d U)\right)^{1 / 2}
\end{aligned}
$$

by the Schwartz inequality, so that for almost all $t$ the measure $\boldsymbol{\lambda}_{\alpha}(t)$ belongs to $C_{\mathrm{b}}(\boldsymbol{U})^{*}=\boldsymbol{\Sigma}_{\mathrm{rba}}\left(\boldsymbol{U}, \boldsymbol{\Phi}_{\mathrm{c}}\right)$ (observe that a similar construction was used in the proof of Lemma ??). It follows from (??) that $t \rightarrow\left\|\boldsymbol{\lambda}_{\alpha}(t)\right\|_{\Sigma_{r b a}\left(U, \Phi_{c}\right)}$ is measurable. Moreover,

$$
\int_{0}^{T}\left\|\boldsymbol{\lambda}_{\alpha}(t)\right\|_{\Sigma_{r b a}\left(U, \Phi_{c}\right)}^{2} d t \leq \int_{0}^{T} \int_{\boldsymbol{U}} \kappa(U)^{2} \mu_{\alpha}(t, d U) d t
$$

and this is uniformly bounded by the hypothesis in Lemma??. Now, since

$$
L^{2}\left(0, T ; C_{\mathrm{b}}(\boldsymbol{U})\right)^{*}=L_{\mathrm{W}}^{2}\left(0, T ; \boldsymbol{\Sigma}_{\mathrm{rba}}\left(\boldsymbol{U}, \boldsymbol{\Phi}_{\mathrm{C}}\right)\right),
$$

passing to a generalized subsequence we may assume that $\left\{\boldsymbol{\lambda}_{\alpha}(\cdot)\right\}$ is $L^{2}\left(0, T ; C_{\mathrm{b}}(\boldsymbol{U})\right)$ weakly convergent to $\boldsymbol{\lambda}(\cdot) \in L_{\mathrm{W}}^{2}\left(0, T ; \boldsymbol{\Sigma}_{\mathrm{rba}}\left(\boldsymbol{U}, \boldsymbol{\Phi}_{\mathrm{C}}\right)\right)$. If $t \rightarrow \kappa(\cdot)^{-1} f(t, \cdot)$ is in $L^{2}\left(0, T ; C_{\mathrm{b}}(\boldsymbol{U})\right)$, we have

$$
\int_{0}^{T} \int_{\boldsymbol{U}} f(t, U) \mu_{\alpha}(t, d U) d t
$$




$$
\begin{aligned}
& =\int_{0}^{T} \int_{\boldsymbol{U}} \kappa(U)^{-1} f(t, U) \kappa(U) \mu_{\alpha}(t, d U) d t \\
& =\int_{0}^{T} \int_{\boldsymbol{U}} \kappa(U)^{-1} f(t, U) \lambda_{\alpha}(t, d U) d t \rightarrow \int_{0}^{T} \int_{\boldsymbol{U}} \kappa(U)^{-1} f(t, U) \lambda(t, d U) d t .
\end{aligned}
$$

Thus, it only remains to be shown that $\lambda(\cdot, d U)=\kappa(U) \mu(\cdot, d U)$, or $\mu(\cdot, d U)=$ $\kappa(U)^{-1} \lambda(\cdot, d U)$. To see this, take $f(t, \cdot)=f(\cdot) \in C_{\mathrm{b}}(\boldsymbol{U})$ and use (??), taking limits directly in the first term. The result is

$$
\int_{0}^{T} \int_{\boldsymbol{U}} f(U) \mu(t, d U) d t=\int_{0}^{T} \int_{\boldsymbol{U}} f(U) \kappa(U)^{-1} \lambda(t, d U) d t
$$

Proposition 5 [?] Let the semigroup $S(t)$ generated by $-\mathcal{A}$ be compact for $t>0$, and let $1 \leq p<\infty$.

(I) Let $1-1 / p \leq \gamma<1$. Then the operator $\Lambda$ defined by

$$
[\Lambda \boldsymbol{g}](t)=\int_{0}^{t} \mathcal{A}^{\gamma} S(t-r) \boldsymbol{g}(r) d r
$$

is a compact operator from $L^{p}(0, T ; \boldsymbol{X})$ into $L^{r}(0, T ; \boldsymbol{X})$ (for any $r<p /[1-$ $p(1-\gamma)]$, for any $r<\infty$ if $\gamma=1-1 / p)$.

(II) Let $0 \leq \gamma<1-1 / p$. Then the operator $\Lambda$ is compact from $L^{p}(0, T ; \boldsymbol{X})$ into $C([0, T] ; \boldsymbol{X})$.

We note that $\Lambda$ is a bounded operator from $L^{2}(0, T ; \boldsymbol{X})$ into $C([0, T] ; \boldsymbol{X})$, but it is not compact.

Existence Theorem 1 and Corollary ?? below are our fundamental existence theorems.

Existence Theorem 1 Assume that (??) holds, $\Theta(\boldsymbol{v}, U) \geq 0$ and that there exists a minimizing sequence $\left\{\boldsymbol{\mu}_{n}(\cdot)\right\}, \boldsymbol{\mu}_{n}(\cdot) \in \mathcal{V}_{b c}\left(0, t_{n} ; \boldsymbol{U}, \kappa\right)$ of chattering controls such that

(a) $\left\{t_{n}\right\}$ is bounded.

$$
\text { (b) }\left\|\boldsymbol{\kappa}\left(\boldsymbol{\mu}_{n}(\cdot)\right)^{2}\right\|_{L^{1}\left(0, t_{n}\right)}=\left\|\int_{\boldsymbol{U}} \kappa(U)^{2} \mu_{n}(\cdot, d U)\right\|_{L^{1}\left(0, t_{n}\right)}
$$

is uniformly bounded.

(c) Each trajectory $\boldsymbol{v}\left(\cdot, \boldsymbol{\mu}_{n}\right)$ exists in $0 \leq t \leq t_{n}$, 